\author{
Szymon Chlebowski \\ Dorota LESZCZYŃSKA-JASION
}

\section{Dual Erotetic Calculi and the Minimal LFI}

\begin{abstract}
An erotetic calculus for a given logic constitutes a sequent-style prooftheoretical formalization of the logic grounded in Inferential Erotetic Logic (IEL). In this paper, a new erotetic calculus for Classical Propositional Logic (CPL), dual with respect to the existing ones, is given. We modify the calculus to obtain complete proof systems for the propositional part of paraconsistent logic CLuN and its extensions CLuNs and mbC. The method is based on dual resolution. Moreover, the resolution rule is non-clausal. According to the authors knowledge, this is the first account of resolution for mbC. Last but not least, as the method is grounded in IEL, it constitutes an important tool for the so-called question-processing.
\end{abstract}

Keywords: Inferential Erotetic Logic, Proof theory of paraconsistent logics, mbC, CLuN, CLuNs, Dual resolution.

\title{
Introduction
}

The considerations presented in this paper join two issues, each of which is of indepedendent interest. First, our work contributes to the proof-theory of paraconsistent logics known as CLuN, CLuNs and $\mathrm{mbC}$. Since $\mathrm{mbC}$ is a basic Logic of Formal Inconsistency (LFI for short), our work is at the same time a contribution to the proof-theory of LFI. Secondly, and probably more importantly, the results presented in this paper constitute a part of a larger project conducted on the grounds of the logic of questions, originated and developed by Andrzej Wiśniewski, and called Inferential Erotetic Logic (IEL).

IEL is a logic which focuses its attention on, by and large, inferences whose conclusions are questions. IEL addresses many issues which are commonly considered under the heading "logic of questions", like typologies of questions, the structure of questions, or the answerhood problem. The distinguishing feature of IEL, however, is that it defines the criteria of the validity of inferences in which questions play the role of a conclusion and,

Presented by Andrzej Indrzejczak; Received June 30, 2014 
possibly, a premise. Inferences of this kind are called erotetic inferences. As an example of an erotetic inference let us present the following ([27, p. 52]):

- Andrew always comes on time, but now he is late. What has happened to him?

Consider also:

- Is the Twin Prime Conjecture true? $n$ is a prime. Is $n-2$ or $n+2$ a prime as well?

- Set $\Gamma$ contains a contradiction. Does it explode on the grounds of logic $\mathrm{L}$, that is, can I derive arbitrary $A$ from $\Gamma$ by means of a deductive apparatus of $\mathrm{L}$ ?

In each of this cases a declarative premise, or a question together with a declarative premise, leads one to arrive at a(nother) question. IEL aims at describing at least some categories of such erotetic inferences and explicating the criteria of their validity. Indeed, it successfully does so - see $[25,27]$.

Recently, the tools of IEL have been used for modelling the phenomenon of internal question processing. Let us quote the source [27, p. 103]:

When an agent is supposed to answer a question or solve a problem, but he/she cannot accomplish the task by means of informational resources which are directly accessible to him/her, it often happens that he/she internally processes the initial question (emphasis added-SzCh, DLJ). The outcome is either a new question concerning the subject matter or a preliminary strategy of reducing the initial question into auxiliary questions. In both cases erotetic inferences play a substantial role. When answers to questions raised are still inaccessible, the process goes further in an analogous way, possibly with the help of data just collected.

Certain types of phenomenon described in the quote may be successfully modelled by means of erotetic calculi. These are basically calculi of questions, where each rule of inference (erotetic rule of inference) processes a question into another question $[26,28,30]$. From a proof-theoretical perspective erotetic calculi are based on inverted sequent calculi, ${ }^{1}$ which may be also viewed as hypersequent systems (with hypersequents joined conjunctively, cf. [1]). The sequents are interpreted as representing meta-level statements

\footnotetext{
${ }^{1}$ See [16] and [12, pp. 1307-1308] for a discussion on the relation between erotetic calculi and more standard sequent calculi. See also $[18,24]$ for background on sequent systems.
} 
concerning an underlying logic, and the questions of a formal language represent questions concerning the statements. The rules of erotetic calculi are constructed in a way which enables the "decomposition" of a question or, in other words, a simplification of the logical structure of the considered problem. Each erotetic calculus has a set of "basic sequents" which express some basic facts concerning the underlying logic. Consequently, a question concerning basic sequents is regarded as "rhetorical", since the answer to it is obvious.

The method of transforming questions (of a certain formal language) concerning some chosen properties of an underlying logic $L$ in accordance with the rules of an erotetic calculus is called the method of Socratic proofs for $L$ (the "Socratic" aspect of the method is concerned with the fact that one aims at questions to which an answer is obvious). If the questions are constructed in such a way that they concern the consequence relation in $\mathrm{L}$, then the method becomes in fact a proof-method for $\mathrm{L}$. The method has been adjusted to CPL [26], to First-Order Logic [28], to the propositional parts of CLuN and CLuNs [30], to a large class of propositional modal logics [13-15], and to propositional intuitionistic logic [21]. Yet still, an erotetic calculus remains a calculus of questions. Using semantic concepts defined on the grounds of Minimal Erotetic Semantics (see [27]) it can be shown that each transition from a question to a question guided by an erotetic rule is an example of a valid erotetic inference in the sense of IEL. These ideas have been developed by Andrzej Wiśniewski and described extensively in [27]. In Sect. 8 we also provide some technical details concerning Minimal Erotetic Semantics for the proposed dual erotetic calculi.

A proof of a sequent ' $\vdash A$ ' in an erotetic calculus is a sequence of questions, called Socratic transformation, with the following properties. It starts with a question of the form: ? $\vdash A)$, intuitively interpreted as concerning the validity or theoremhood of formula $A$. Each question from that sequence is obtained from the previous question by means of an erotetic rule of inference, which "decomposes" or "simplifies" a question on which it acts. Last but not least, the affirmative answer to the last question is in a sense evident, and the construction of erotetic rules warrants that from the fact that the answer to the last question of a Socratic transformation is affirmative it follows that the answer to the initial question? $(\vdash A)$ is affirmative.

Generally speaking, if the erotetic calculi considered so far are calculi of questions concerning validity, then the dual erotetic calculi are calculi of questions concerning refutability. The notion of refutability is to be understood as proving satisfiability of the converse, or proving falsifiability in 
exactly the sense known from the resolution systems. The dual erotetic calculi differ from the existing ones primarily in the following aspect. The rules of dual erotetic calculi decompose a formula to its disjunctive normal form (DNF), whereas in original erotetic calculi the method of decomposition reflects the deriving conjunctive normal form (CNF) of a formula. And, as should be expected, the resolution rule is indispensible in the derivation process. $^{2}$

Now to the logics we have chosen for the dual erotetic description.

CLuN, introduced in [2], is a predicative paraconsistent logic. It is the weakest negation-complete extension of positive classical logic. CLuNs is a very rich extension of CLuN which remains paraconsistent although its axioms allow us to introduce the paraconsistent negation inside formulas (see [4]). Both logics are used for the construction of inconsistency-adaptive logics (see e.g. [3]). In this paper we shall consider only the propositional fragments of these logics, we will also omit the equivalence connective. For simplicity's sake, we use the names CLuN and CLuNs for the propositional fragments considered here. ${ }^{3}$

LFI are paraconsistent logics which "internalize" the property of consistency (and/or inconsistency) expressing it as an operator, and thus allowing us to recover all of Classical Logic inside the system (see [7]). Logic mbC is the minimal LFI based on Classical Logic. At the same time, mbC may be thought of as an extension of CLuN (see $[4,5]$ ). Logic mbC has been introduced in [8] and has gained popularity over the decade. As to the prooftheoretical descriptions, there is a tableau method for $\mathrm{mbC}$ [7], the $\mathbf{K E}$ tableau method, which is also implemented [19], and there is also a sequent system for $\mathrm{mbC}[9]$. There is no resolution-account of $\mathrm{mbC}$ known to us.

It seems that erotetic inferences may be paraconsistent as well, thus the philosophical reason for the construction presented in this paper is that embedding paraconsistent logics into the erotetic framework results in a

\footnotetext{
${ }^{2}$ Interestingly, since we deal with sequents, the resolution rule could be viewed as a form of the cut-rule, which changes the dual erotetic calculi into sequent calculi with a structural rule of cut, which cannot be eliminated. Admissible structural rules of erotetic calculi were also considered, see [29].

${ }^{3}$ Strictly speaking, we are considering certain linguistic extensions of (propositional) systems CLuN, CLuNs and mbC. In each of these systems we have two kinds of negations: the classical and the paraconsitent one. There are two main reasons for considering such extensions. Firstly, on the grounds of LFl's we can observe interesting dependencies between the consistency operator, the paraconsistent and the classical negation. Secondly, the rule of resolution works in a more transparent way when we use simply classical negation instead of, for example, some kind of labels or signed formulas.
} 
formal model of erotetic inferences in which contradiction appears. However, the chief motivation for considering CLuN and CLuNs here is that these logics had already been considered in an erotetic setting in [30]. This opens up an interesting opportunity to examine the duality relation between the constructed systems.

In Sect. 5 we present a sound and complete resolution system for CPL, CLuN, CLuNs and mbC. The system is based on a special form of dual resolution. Moreover, it is non-clausal. (For a detailed explanation see Sect. 1.1.) Incidentally, it follows from this result that each of these paraconsistent logics has intuitive normal forms which may be, however, expressed only in a language richer than the standard object-level language of the given logic. A similar technique is used in [6] in the context of embedding logic CLuN in $\mathrm{CPL}$, where the normal form of a formula is expressed in a language with a new kind of variables.

\section{Why Dual Resolution?}

\subsection{A Note on Dual Resolution and Non-clausal Resolution}

Let $\mathcal{L}_{\mathrm{CPL}}$ be the language of CPL. For each propositional formula $A$ of $\mathcal{L}_{\mathrm{CPL}}$ we define $A^{\mathbf{n}}$ and $A^{\mathbf{d}}$ as follows:

- $A^{\mathbf{n}}$ is the result of replacing each occurrence of a negated propositional variable, $\neg p_{i}$, with $p_{i}$, and each occurrence of a propositional variable, $p_{i}$, which is not an argument of negation, with its negation $\neg p_{i}$.

- $\quad A^{\mathrm{d}}$ is the result of replacing each occurrence of a binary connective with an occurrence of its dual, e.g. ' $\wedge$ ' is replaced with ' $\vee$ ' and conversely.

Then it is a well-known result that for each Boolean valuation $v$ :

$$
v\left(\neg\left(A^{\mathbf{n}}\right)\right)=v\left(A^{\mathbf{d}}\right)
$$

for each formula $A$ of $\mathcal{L}_{\mathrm{CPL}}$ (see for example [10, Chap. 2]), which amounts to the fact that:

$$
\neg\left(A^{\mathbf{n}}\right) \leftrightarrow A^{\mathbf{d}}
$$

is a law of CPL, but this yields that also ' $\neg \neg\left(A^{\mathbf{n}}\right) \leftrightarrow \neg\left(A^{\mathbf{d}}\right)$ ' is a law of CPL, and this entails that:

$$
\left(\neg \neg\left(A^{\mathbf{n}}\right)\right)^{\mathbf{d}} \leftrightarrow\left(\neg\left(A^{\mathbf{d}}\right)\right)^{\mathbf{d}}
$$

is a law of CPL, which, as is easy to establish, yields that so is:

$$
\left(A^{\mathbf{n}}\right)^{\mathbf{d}} \leftrightarrow \neg A
$$


Now suppose that $A$ is in DNF. Then, trivially, the formula $\left(A^{\mathbf{n}}\right)^{\mathbf{d}}$ is in CNF, and by (IV) it is a CNF of $\neg A$. This simple result shows that for applying the rule of resolution it is irrelevant whether one starts with the CNF of $\neg A$ or the DNF of $A$. In the first case we arrive at a standard system, where the resolution rule acts on clauses and preserves their satisfiability. In the second case, we work in the dual system, with the dual resolution rule which acts on dual clauses (conjunctions of literals) and preserves their "falsifiability", understood as the property of being false under some valuation. To complete the picture let us remember that the empty clause is unsatisfiable, since it is an empty disjunction, whereas the empty dual clause, being an empty conjunction, is unfalsifiable.

These nice properties of duality have led us to dual resolution and dual erotetic calculi. In fact, the notion of dual resolution, as a system of resolution starting with the DNF of a formula and using the dual resolution rule, is quite commonly used, see e.g. [17] and its bibliography.

The idea of a "non-clausal resolution" has been described in [10]. The rule of a non-clausal resolution may act on the generalized conjunctions of formulas, which may not be clauses, as they may contain compound formulas. The formula which is "cut" by the resolution rule also may be compound. The system of clausal resolution has all the usual semantic properties of a resolution system but also some additional merits from the point of view of complexity and implementation issues, as it allows us to shorten the process of arriving at the normal form (for more extensive commentary on the non-clausal resolution in the context of non-classical logics see [11, Chap. 3]).

\subsection{An Erotetic Account of Dual Resolution}

Suppose we wonder whether the formula $A=(p \rightarrow q) \rightarrow(\neg q \rightarrow \neg p)$ is refutable in CPL (falsifiable, in more semantic terms). Naturally, this amounts to accepting the antecedent and rejecting the consequent of $A$; if for some reason, however, we wish to establish what is to be refuted, then this brings us to the question of whether one may refute (falsify) the negation of the antecedent together with the consequent, that is, whether these two formulas may be falsified at the same time. If we represent the first question as

$$
?(\dashv(p \rightarrow q) \rightarrow(\neg q \rightarrow \neg p))
$$

where the inverted turnstile ' - ' stands for refutability (or, to turn to semantic terms once again, falsifiability, that is, invalidity of a formula), then we may pass from question $(\mathrm{V})$ to question:

$$
?(\dashv \neg(p \rightarrow q) ; \dashv \neg q \rightarrow \neg p)
$$

and, on the same basis, to: 


$$
?(\dashv \neg(p \rightarrow q) ; \dashv \neg \neg q ; \dashv \neg p)
$$

The semicolon ';' clearly represents a kind of metalevel conjunction, thus we may read question (VII) as "Is it the case that $\neg(p \rightarrow q)$ is refuted and $\neg \neg q$ is refuted and $\neg p$ is refuted at the same time?". Another possible reading is the following: "Is it the case that $\neg(p \rightarrow q)$ is falsified and $\neg \neg q$ is falsified and $\neg p$ is falsified at the same time (that is, false under the same valuation)?" Refuting a negated implication amounts to refuting at least one of the appropriately defined conjuncts, which suggests passing from (VII) to:

$$
?(\dashv p, \neg q ; \dashv q ; \dashv \neg p)
$$

(we have also removed the double negation). The commas separating formulas after the inverted turnstile act as a metalevel disjunction. Thus we read question (VIII) as follows: "Is it the case that at the same time at least one of $p, \neg q$ is refuted, $q$ is refuted, and $\neg p$ is refuted?"

This process of passing from a question to a question is a formal description of the phenomenon of solving a problem (here: falsifiability of a formula) purely by transforming it, that is, with no reference to any declarative premise. At the same time, it reveals an intuitive background for applying resolution in the erotetic setting. Observe that, if at least one of: $p, \neg q$ is refuted and at the same time $q$ is refuted, then (taking the laws of Classical Logic for granted) $p$ must be refuted. In this way we pass to question:

$$
?(\dashv p ; \dashv \neg p)
$$

But refuting $p$ and $\neg p$ at the same time (i.e. falsifying at the same valuation) is not an option. ${ }^{4}$ Therefore (IX) leads us to question whether the empty disjunction may be refuted, which is not the case. We will represent this kind of question in the following way:

$$
?(\dashv \emptyset)
$$

The process of transforming the questions presented above resembles a resolution refutation. Observe that, at any stage of this process, if the answer to a certain question is negative (that is, one cannot refute formula(s) concerned by the question), then the answer to the previous question must be

\footnotetext{
${ }^{4}$ The difference between the notion of refutability considered here and the one known from the tradition of refutation systems (see e.g. [22]) is clear in this context, as one can have $p$ and $\neg p$ refuted in a refutation system (both are non-theses), but one cannot have them both falsified under the same valuation. In other words, the rules of refutation systems preserve non-validity, whereas the rules presented in this paper preserve - as we shall see-falsifiability under a valuation.
} 
negative as well. Moreover, (X) expresses a kind of a "dual Socratic question" to which an answer is plainly negative.

Things are not as straightforward as we would wish, however. Let us observe that the following formula:

$$
(p \wedge \neg q) \vee q \vee \neg p
$$

which may be extracted from question (VIII), is a DNF of formula $A$. It means that it may be more natural to interpret a semicolon as a disjunction and a comma as a conjunction. Under this interpretation, question (V) concerns the validity of $A$. The reading of question (VIII) becomes: "Is it the case that under any valuation either $p$ and $\neg q$ holds or $q$ holds or $\neg p$ holds?" We find the first interpretation (in terms of refutability) more natural when intuitions are concerned, but we actually use the second interpretation (in terms of validity) on formal grounds.

This is not merely a formal trick. The duality of interpretation reflects the dual nature of our reasoning. In order to clarify the matter, we stress that the two interpretations simply pertain to different levels of the analysis of our questions. The interpretation in terms of the validity of a DNF of a formula pertains to the level of the underlying logic, which is the CPL in this case, whereas the interpretation in terms of refutability (falsifiability) of formulas pertains to the level of sequents, which represent meta-statements with respect to the underlying logic. Thus on the level of questions we can say that a negative answer to $(\mathrm{X})$ means that one cannot refute an empty conjunction. The answer to question $(\mathrm{V})$ is thus also negative: one cannot refute the formula considered there, and this amounts to the fact that the formula is CPL-valid.

\section{The Erotetic Language}

Let $\mathcal{L}_{\mathrm{L}}$ denote the language of a certain propositional logic $\mathrm{L}$ (the Classical Logic for example). Then language $\mathcal{L}_{-L}^{\text {? }}$ is an object-level language in which the dual erotetic calculus for $L$ will be worded. The meaningful expressions of $\mathcal{L}_{-\mathrm{L}}^{\text {? }}$ belong to two disjoint sets. The first one consists of declarative well-formed formulas (hereafter $\mathrm{d}$-wffs). The second one is the set of erotetic well-formed formulas (e-wffs, questions). To obtain the vocabulary of $\mathcal{L}_{-\mathrm{L}}^{\text {? }}$ we add to the vocabulary of $\mathcal{L}_{\mathrm{L}}$ the following signs: $\dashv$, ? (a sign

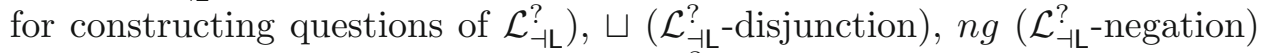
and: , (comma), ; (semicolon). We call $\mathcal{L}_{-\mathrm{L}}^{\text {? }}$ a standard erotetic extension of language $\mathcal{L}_{\mathrm{L}}$. 
An atomic declarative well-formed formula of $\mathcal{L}_{-\mathrm{L}}^{?}$ is an expression of the form:

$$
\dashv S
$$

where $S=\left\langle A_{1}, \ldots, A_{n}\right\rangle$ is a finite (possibly empty) sequence of formulas of $\mathcal{L}_{\mathrm{L}}$. We will write:

$$
\dashv A_{1}, \ldots, A_{n}
$$

instead of: $\dashv\left\langle A_{1}, \ldots, A_{n}\right\rangle$. The expressions of the form (XIII) will be called reversed sequents or simply sequents of $\mathcal{L}_{\dashv L}^{?}$. If $S$ is the empty sequence, then we write ' $S=\emptyset$ ' and we call the sequent ' $\dashv \emptyset$ ' the (reversed) empty sequent. Compound d-wffs of $\mathcal{L}_{-L}^{?}$ are built from atomic d-wffs of $\mathcal{L}_{-L}^{?}$ by means of $\sqcup$ and $n g$. The set $\mathcal{D}_{\mathcal{L}_{-L}^{?}}$ (the set of d-wffs of $\left.\mathcal{L}_{-L L}^{?}\right)$ is the smallest among sets $\mathcal{D}$ satisfying the following conditions: (i) every atomic d-wff is in $\mathcal{D}$, (ii) if $X$ is in $\mathcal{D}$, then $n g(X)$ is in $\mathcal{D}$, (iii) if $X, Y$ are in $\mathcal{D}$ then $(X \sqcup Y)$ is in $\mathcal{D}$.

Questions of $\mathcal{L}_{\dashv L}^{?}$ are expressions of the following form:

$$
?(\Phi)
$$

where $\Phi$ is a finite and non-empty sequence of atomic d-wffs of $\mathcal{L}_{\dashv L}^{\text {? }}$, i.e. reversed sequents of $\mathcal{L}_{-\mathrm{L}}^{\text {? }}$. A question of the form (XIV) is said to be based on the sequence $\Phi$. Terms of the sequence $\Phi$ are said to be constituents of the question (XIV). We will use symbols $\phi, \psi$ (possibly with subscripts) as metalinguistic variables for reversed sequents and symbols $Q, Q^{\prime}, Q^{*}$ (possibly with subscripts) as metalinguistic variables for questions.

Let $\Phi=\left\langle\phi_{1}, \ldots, \phi_{n}\right\rangle$ be a sequence of sequents of $\mathcal{L}_{-\mathrm{L}}^{?}$, and let $Q=?(\Phi)$ be a question of $\mathcal{L}_{\dashv \mathrm{L}}$. Then the following d-wffs:

$$
\begin{aligned}
& \operatorname{af} Q=\left(\phi_{1} \sqcup\left(\phi_{2} \sqcup \ldots \sqcup\left(\phi_{n-1} \sqcup \phi_{n}\right) \ldots\right)\right) \\
& \operatorname{ng} Q=n g\left(\phi_{1} \sqcup\left(\phi_{2} \sqcup \ldots \sqcup\left(\phi_{n-1} \sqcup \phi_{n}\right) \ldots\right)\right)
\end{aligned}
$$

are the affirmative answer to $Q$ and the negative answer to $Q$ respectively. Thus all questions of $\mathcal{L}_{\dashv \mathrm{L}}^{\text {? }}$ are polar questions.

We will use the symbol "' as a concatenation sign for sequences of d-wffs of $\mathcal{L}_{\mathrm{L}}$. Thus ' $S$ ' $T$ ' is the resulting concatenation of the sequences $S$ and $T$. When dealing with one-term sequences we will write ' $S$ ' $A^{\prime}$ ' $T$ ' rather than ' $S$ ' $\langle A\rangle^{\prime} T$ '. For a concatenation of sequences of sequents we will use the semicolon ' $;$ '. Hence we will write ' $\Phi$; $\Psi$ ' for the concatenation of $\Phi$ and $\Psi$. We will also use the semicolon to separate single sequents. 


\section{The Paraconsistent Logics CLuN, CLuNs and mbC}

\subsection{CLuN}

The language $\mathcal{L}_{\text {CLuN }}$ of CLuN consists of the following signs: the set VAR of propositional variables, $\wedge$ (conjunction $), \vee($ disjunction $), \rightarrow($ implication $)$, and the two negations: $\neg$ (classical negation $), \sim$ (paraconsistent negation $)$. The set FOR ${ }^{\mathrm{CLuN}}$ of formulas of $\mathcal{L}_{\mathrm{CLuN}}$ is defined by the following grammar:

$$
A, B::=\operatorname{VAR}|\neg A| \sim A|A \wedge B| A \vee B \mid A \rightarrow B
$$

Let us stress that we consider a $\neg$-extension of the original system CLuN (cf. $[2])$.

Table 1 presents the Hilbert-style deductive system for CLuN. Axiom 12 can be equivalently stated as follows: $(A \rightarrow \sim A) \rightarrow \sim A$.

Definition 3.1. (CLuN-semivaluation) A CLuN-semivaluation is a function $v: \mathrm{FOR}^{\mathrm{CLuN}} \longrightarrow\{0,1\}$ which satisfies the following conditions:

$(v 1) v(A \wedge B)=1$ iff $v(A)=v(B)=1$

(v2) $v(A \vee B)=0$ iff $v(A)=v(B)=0$

$(v 3) v(A \rightarrow B)=0$ iff $v(A)=1$ and $v(B)=0$

(v4) $v(\neg A)=1$ iff $v(A)=0$

$(v 5)$ if $v(\sim A)=0$, then $v(A)=1$

Clause (v5) is not an equivalence. The fact that $v(A)=1$ does not determine the logical value of $\sim A$ : either $v(\sim A)=1$ or $v(\sim A)=0$. In the present proof-theoretical context it is more convenient to make use of the notion of $\mathrm{CLuN}$-valuation:

Definition 3.2. (CLuN-valuation) Let FOR ${ }_{\sim}^{\mathrm{CLuN}}$ denote the set of all paraconsistently negated formulas of $\mathcal{L}_{\mathrm{CLuN}}$. A function $\sigma: \mathrm{FOR}^{\mathrm{CLuN}} \longrightarrow\{0,1\}$

Table 1. The axiom system for CLuN
(1) $A \rightarrow(B \rightarrow A)$
(2) $(A \rightarrow B) \rightarrow(((A \rightarrow(B \rightarrow C)) \rightarrow(A \rightarrow C))$
(3) $A \rightarrow(B \rightarrow(A \wedge B))$
(4) $(A \wedge B) \rightarrow A$
(5) $(A \wedge B) \rightarrow B$
(6) $A \rightarrow(A \vee B)$
(7) $B \rightarrow(A \vee B)$
(8) $(A \rightarrow C) \rightarrow((B \rightarrow C) \rightarrow(A \vee B \rightarrow C))$
(9) $A \vee(A \rightarrow B)$
(10) $A \vee \neg A$
(11) $A \rightarrow(\neg A \rightarrow B)$
(12) $A \vee \sim A$
(MP) If $\vdash_{\text {CLuN }} A$ and $\vdash_{\text {CLuN }} A \rightarrow B$, then $\vdash_{\text {CLuN }} B$ 
Table 2. The axioms for the CLuNs-negation

\begin{tabular}{llll}
\hline $\mathrm{A}(\sim \sim 1)$ & $\sim \sim A \rightarrow A$ & $\mathrm{~A}(\sim \sim 2)$ & $A \rightarrow \sim \sim A$ \\
$\mathrm{~A}(\sim \rightarrow 1)$ & $\sim(A \rightarrow B) \rightarrow A \wedge \sim B$ & $\mathrm{~A}(\sim \rightarrow 2)$ & $A \wedge \sim B \rightarrow \sim(A \rightarrow B)$ \\
$\mathrm{A}(\sim \wedge 1)$ & $\sim(A \wedge B) \rightarrow \sim A \vee \sim B$ & $\mathrm{~A}(\sim \wedge 2)$ & $\sim A \vee \sim B \rightarrow \sim(A \wedge B)$ \\
$\mathrm{A}(\sim \vee 1)$ & $\sim(A \vee B) \rightarrow \sim A \wedge \sim B$ & $\mathrm{~A}(\sim \vee 2)$ & $\sim A \wedge \sim B \rightarrow \sim(A \vee B)$ \\
\hline
\end{tabular}

is CLuN-valuation iff $\sigma$ behaves classically in the case of positive connectives and there exists an assignment function $\bar{\sigma}: \mathrm{FOR}_{\sim}^{\mathrm{CLuN}} \longrightarrow\{0,1\}$ for paraconsistently negated formulas of $\mathcal{L}_{\mathrm{CLuN}}$ such that the following condition holds:

(1) $\sigma(\sim A)=1$ iff $\sigma(A)=0$ or $\bar{\sigma}(\sim A)=1$

Thus $\bar{\sigma}$ directly assigns a logical value to paraconsistently negated formulas independently of the value assigned by $\sigma$ to the formulas. For further reference let us also observe that:

$\left(1^{*}\right) \sigma(\sim A)=0$ iff $\sigma(A)=1$ and $\bar{\sigma}(\sim A)=0$

The two definitions: that of CLuN-semivaluation and that of CLuNvaluation, result in equivalent accounts of CLuN. A formula $A$ of $\mathcal{L}_{\mathrm{CLuN}}$ is true under each CLuN-semivaluation iff $A$ is true under each CLuN-valuation. ${ }^{5}$

\subsection{CLuNs}

The language of CLuNs is the same as that of CLuN, and the set of formulas of $\mathcal{L}_{\text {CLuns }}$ equals the set of formulas of $\mathcal{L}_{\text {CLuN }}$.

CLuNs is a paraconsistent logic with nice properties which allow us to "draw" the paraconsistent negation "inside a formula". The properties are expressed by the axioms for CLuNs-negation (displayed in Table 2) and also by clauses $(v 6)-(v 9)$ of Definition (3.3) below.

Definition 3.3. (CLuNs-semivaluation). To clauses $(v 1)-(v 4)$ of the definition of CLuN-semivaluation we add the following conditions:

$\left(v 5^{\prime}\right)$ if $v(\sim A)=0$, then $v(A)=1$, where $A \in$ VAR or $A=\neg B$ for some $B$ (v6) $v(\sim(A \vee B))=v(\sim A \wedge \sim B)$

\footnotetext{
${ }^{5}$ The notion of CLuN-valuation is the basis of the standard approach to the semantics of CLuN $[4,5]$. The concept of CLuN-semivaluation is not explicitly analysed in the literature on CLuN and CLuNs, but the idea of semivaluation is present in the studies on LFIs [7]. To make semantic intuitions clearer, we describe, for each analysed logic $L$, the concepts of L-semivaluation and L-valuation.
} 
(v7) $v(\sim(A \rightarrow B))=v(A \wedge \sim B)$

$(v 8) v(\sim(A \wedge B))=v(\sim A \vee \sim B)$

(v9) $v(\sim \sim A)=v(A)$

The notion of CLuNs-valuation is akin to the notion of CLuN-valuation. The difference lies in the form of formulas which receive the direct $\bar{\sigma}$-assignment: they must be of the form $\sim A$, where either $A \in \mathrm{VAR}$ or $A$ has the form $\neg B$ for some $B$. Moreover, the clauses analogous to clauses $(v 6)-(v 9)$ hold for CLuNs-valuation.

\section{3. $\mathrm{mbC}$}

The language $\mathcal{L}_{\mathrm{mbC}}$ of the logic $\mathrm{mbC}$ is the language of CLuN enriched with the symbol o (consistency operator). The set $\mathrm{FOR}^{\mathrm{mbC}}$ of formulas of $\mathcal{L}_{\mathrm{mbC}}$ is defined by the following grammar:

$$
A, B::=\operatorname{VAR}|\neg A| \sim A|\circ A| A \wedge B|A \vee B| A \rightarrow B
$$

The logic $\mathrm{mbC}$ is usually worded in a language without classical negation, but it is possible to define the constant falsum in it by putting:

$$
\perp::=\circ A \wedge(A \wedge \sim A)
$$

Then the classical negation is defined by: $\neg A::=A \rightarrow \perp$. However, as we consider $\mathrm{mbC}$ as an extension of CLuN we take $\neg$ as primitive.

In order to obtain the axiomatic characterization of $\mathrm{mbC}$ we add the following axiom to the axioms of CLuN:

$\operatorname{Ax}(\circ) \quad \circ A \rightarrow(A \rightarrow(\sim A \rightarrow B))$

DeFinition 3.4. (mbC-semivaluation $\left.{ }^{6}\right)$. An mbC-semivaluation is a function $v: \mathrm{FOR}^{\mathrm{mbC}} \longrightarrow\{0,1\}$ which satisfies the conditions posed on CLuNvaluations plus the following clause:

(०) if $v(\circ A)=1$, then $v(A)=0$ or $v(\sim A)=0$.

As in the case of CLuN and CLuNs it is more convenient to work with the notion of $\mathrm{mbC}$-valuation instead of semivaluation:

DEFINITION 3.5. (mbC-valuation). Let $\mathrm{FOR}_{\sim \circ}^{\mathrm{mbC}}$ denote the set of all formulas of $\mathrm{FOR}^{\mathrm{mbC}}$ of the form ' $\sim A$ ' or ' $\circ A$ '. A function $\lambda: \operatorname{FOR}^{\mathrm{mbC}} \longrightarrow\{0,1\}$ is $\mathrm{mbC}$-valuation iff $\lambda$ behaves classically in the case of positive connectives

\footnotetext{
${ }^{6}$ The notion of $\mathrm{mbC}$-semivaluation is described in [7] (but under the name "bivaluation semantics for $m b C ")$.
} 
and there exists an assignment function $\bar{\lambda}: \mathrm{FOR}_{\sim \circ}^{\mathrm{mbC}} \longrightarrow\{0,1\}$ such that the following conditions hold:

(1) $\lambda(\sim A)=1$ iff $\lambda(A)=0$ or $\bar{\lambda}(\sim A)=1$

(2) $\lambda(\circ A)=1$ iff $(\lambda(A)=0$ and $\bar{\lambda}(\circ A)=1)$ or $(\lambda(\sim A)=0$ and $\bar{\lambda}(\circ A)=1)$

For further reference let us state a truth condition for formulas of the form ' $\neg \circ A$ ':

$$
\lambda(\circ A)=0 \text { iff } \lambda(A)=\lambda(\sim A)=1 \text { or } \bar{\lambda}(\circ A)=0
$$

\section{Sequents and Semantics}

Now let us go back to the core idea of this paper - the erotetic account of the dual resolution systems for the presented logics.

\subsection{Erotetic Rules of Inference}

Each erotetic rule of inference consists of a premise and a conclusion which are both questions. Each question is based on a finite sequence of reversed sequents. The rules of the calculus $\mathbb{E}_{\text {res }}^{\mathrm{L}}$ take the following forms:

$$
\frac{?(\Phi ; \phi ; \Psi)}{?\left(\Phi ; \phi^{\prime} ; \Psi\right)} \mathbf{R}_{n b} \frac{?(\Phi ; \phi ; \Psi)}{?\left(\Phi ; \phi^{\prime} ; \phi^{\prime \prime} ; \Psi\right)} \mathbf{R}_{b} \frac{?(\Phi ; \phi ; \Psi ; \psi ; \Omega)}{?\left(\phi^{\prime} ; \Phi ; \Psi ; \Omega ; \phi ; \psi\right)} \mathbf{R}_{b b}
$$

We call the sequence ' $\Phi ; \phi ; \Psi$ ' (respectively, ' $\Phi ; \phi ; \Psi ; \psi ; \Omega$ ') the premise sequence of a given rule. Sequence ' $\Phi ; \phi^{\prime} ; \Psi^{\prime}$ (respectively, ' $\Phi ; \phi^{\prime} ; \phi^{\prime \prime} ; \Psi$ ' or ' $\phi$ ' $\Phi ; \Psi ; \Omega ; \phi ; \psi$ ') is called the conclusion sequence of a given rule.

Sequents $\phi$ (and $\psi$ ) distinguished in the schema of rule $\mathbf{R}=\mathbf{R}_{b}, \mathbf{R}_{n b}$, $\mathbf{R}_{b b}$ is (are) called active sequent (s) or premise sequent $(s)$ of $\mathbf{R}$. Similarly, sequent $\phi^{\prime}$ (sequents $\phi^{\prime}$ and $\phi^{\prime \prime}$ ) is (are) called conclusion sequent $(s)$ of $\mathbf{R}$. The aim of the $\mathbf{R}_{b}$ or $\mathbf{R}_{n b}$ rule is to decompose a formula. A formula occurring in the active sequent of a rule $\mathbf{R}$ such that some connective of that formula is eliminated by the rule is an active formula of that rule. We will also say that sequent $\phi^{\prime}$ distinguished in the schema of $\mathbf{R}_{\mathbf{b b}}$ is obtained from the active sequents $\phi$ and $\psi$.

Rules $\mathbf{R}_{b}$ and $\mathbf{R}_{n b}$ are called eliminative. The eliminative rules of our systems are the dual (and erotetic) counterparts of the Resolution Expansion Rules which are used to derive CNF in the system of the non-clausal resolution presented in [10]. 


\subsection{Reversed Sequents}

Now we pass to the formal semantic interpretation of erotetic wffs. Henceforth let $\mathrm{L} \in\{\mathrm{CPL}, \mathrm{CLuN}, \mathrm{CLuNs}, \mathrm{mbC}\}$, and let $\mathcal{L}_{\mathrm{L}}$ denote the language of $\mathrm{L}$.

Recall that according to the intuitions presented in Sect. 1.2, a sequent of the form (XIII): $\dashv A_{1}, \ldots, A_{n}$ represents the following meta-statement (meta- with respect to $\mathrm{L}$ ): "under the present circumstances (i.e. valuation) at least one of $A_{1}, \ldots, A_{n}$ is refuted (i.e. falsified)". Taking the intuitions for granted, we wish to evaluate the sequent as correct (under a valuation), if the statement represented by the sequent is true. This amounts to the fact that one of $A_{1}, \ldots, A_{n}$ is false (under this valuation). Thus we can say that sequent (XIII) is incorrect under an L-valuation $V$ iff $V\left(A_{1}\right)=\cdots=$ $V\left(A_{n}\right)=1$. Since ultimately a logician is interested in truth rather than non-truth, the pivotal semantic notion pertaining to sequents happens to be that of being incorrect, so as a result we can find a counterpart of validity by establishing that the sequent of the form: $\dashv A$ is incorrect under any $\mathrm{L}-$ valuation iff $A$ is $\mathrm{L}$-valid. This is the price one pays for playing with double negation, so to say.

Definition 4.1. Let $S=\left\langle A_{1}, \ldots, A_{n}\right\rangle$ be a sequence of formulas of $\mathcal{L}_{\mathrm{L}}$. We say that a reversed sequent $\dashv S$ is incorrect under L-valuation $v$, if for every $1 \leq i \leq n, v\left(A_{i}\right)=1$, otherwise we say that $\dashv S$ is correct under $v$.

Quite trivially:

Corollary 4.2. For each $\mathrm{L}$ :

1. for each $\mathrm{L}$-valuation $v$, the empty sequent $\dashv \emptyset$ is incorrect under $v$,

2. sequent $\dashv A$ is incorrect under every $\mathrm{L}$-valuation $v$ iff $A$ is $\mathrm{L}$-valid.

Clause 2. of Corollary 4.2 justifies us in saying that the answer to question: ? $(\dashv A)$, concerning the refutability of $A$, is negative iff $A$ is not refutable, i.e. it is valid.

Definition 4.3. Let $\Phi=\left\langle\phi_{1} ; \ldots ; \phi_{n}\right\rangle$ be a sequence of sequents of $\mathcal{L}_{-\mathrm{L}}^{\text {? }}$ and let $v$ be an arbitrary L-valuation. We say that $\Phi$ is incorrect under $v$, if at least one sequent $\phi_{i}(1 \leq i \leq n)$ is incorrect under $v$, and otherwise we say that $\Phi$ is correct under $v$.

By Corollary 4.2 and Definition 4.3 we arrive at:

Corollary 4.4. If $\dashv \emptyset$ is a term of sequence $\Phi$, then $\Phi$ is incorrect under each L-valuation $v$.

Finally, concerning the interpretation in terms of the DNF of a formula mentioned above, let us note that by Definition 4.1 and 4.3 : 
Table 3. $\alpha / \beta$ notation

\begin{tabular}{cccccc}
\hline$\alpha$ & $\alpha_{1}$ & $\alpha_{2}$ & $\beta$ & $\beta_{1}$ & $\beta_{2}$ \\
\hline$A \wedge B$ & $A$ & $B$ & $\neg(A \wedge B)$ & $\neg A$ & $\neg B$ \\
$\neg(A \vee B)$ & $\neg A$ & $\neg B$ & $A \vee B$ & $A$ & $B$ \\
$\neg(A \rightarrow B)$ & $A$ & $\neg B$ & $A \rightarrow B$ & $\neg A$ & $B$ \\
\hline
\end{tabular}

Table 4. Rules of $\mathbb{E}_{\text {res }}^{\mathrm{CPL}}$

$$
\begin{gathered}
\frac{?\left(\Phi ; \dashv S^{\prime} \beta^{\prime} T ; \Psi\right)}{?\left(\Phi ; \dashv S^{\prime} \beta_{1}{ }^{\prime} T ; \dashv S^{\prime} \beta_{2}{ }^{\prime} T ; \Psi\right)} \mathbf{R}_{\beta} \\
\frac{?\left(\Phi ; \dashv S^{\prime} \alpha^{\prime} T ; \Psi\right)}{?\left(\Phi ; \dashv S^{\prime} \alpha_{1}{ }^{\prime} \alpha_{2}{ }^{\prime} T ; \Psi\right)} \mathbf{R}_{\alpha} \\
?\left(\Phi ; \dashv S^{\prime} \neg \neg A^{\prime} T ; \Psi\right) \\
\frac{?\left(\Phi ; \dashv S^{\prime} A^{\prime} T ; \Psi ; \dashv U^{\prime} \bar{A}^{\prime} V ; \Omega\right)}{?\left(\dashv \underline{S}^{\prime} \underline{T}^{\prime} \underline{U}^{\prime} \underline{V} ; \Phi ; \Psi ; \Omega ; \dashv S^{\prime} A^{\prime} T ; \dashv U^{\prime} \bar{A}^{\prime} V\right)} \mathbf{R}_{\text {res }} \\
\hline
\end{gathered}
$$

Corollary 4.5. For $1 \leq i \leq n$ let $S_{i}=\left\langle A_{j}^{i}\right\rangle_{1 \leq j \leq k_{i}}$ be sequences of formulas of $\mathcal{L}_{\mathrm{L}}$ and let $v$ be an L-valuation. Sequence $\left\langle\dashv S_{1} ; \cdots ; \dashv S_{n}\right\rangle$ is incorrect under $v$ iff $v\left(\left(A_{1}^{1} \wedge \cdots \wedge A_{k_{1}}^{1}\right) \vee \cdots \vee\left(A_{1}^{n} \wedge \cdots \wedge A_{k_{n}}^{n}\right)\right)=1$.

Hence we can say that under all circumstances the answer to question ? $(\dashv$ $\left.S_{1} ; \ldots ; \dashv S_{n}\right)$ is negative iff the formula $\left(A_{1}^{1} \wedge \cdots \wedge A_{k_{1}}^{1}\right) \vee \cdots \vee\left(A_{1}^{n} \wedge \cdots \wedge A_{k_{n}}^{n}\right)$ is valid. Thus Corollary 4.5 supports our intuitive account of questions as questions concerning refutability of formulas.

\section{Erotetic Calculi}

Following Smullyan [23] we have made use of the $\alpha / \beta$ notation (see Table 3 ).

\subsection{Erotetic Calculus for CPL}

The erotetic rules of the calculus $\mathbb{E}_{\text {res }}^{\mathrm{CPL}}$ for CPL are displayed in Table 4 . The resolution rule $\mathbf{R}_{\text {res }}$ may be applied provided that $A$ and $\bar{A}$ are complementary, that is, either $A=\neg \bar{A}$ or $\bar{A}=\neg A$.

For future reference let us set up what follows. If $A=\neg \bar{A}$, then by $\overline{\bar{A}}$ we mean $\bar{A}$, and otherwise by $\overline{\bar{A}}$ we mean $A$. We will say that sequents $\dashv S^{\prime} A^{\prime} T$ and $\dashv U^{\prime} \bar{A}^{\prime} V$ collide on the complementary formulas $A$ and $\bar{A}$, or that they collide on $\overline{\bar{A}}$. We will also say that rule $\mathbf{R}_{\text {res }}$ is applied with respect to $\overline{\bar{A}}$.

The sequences $\underline{S}$ and $\underline{T}(\underline{U}$ and $\underline{V})$ are obtained from sequences $S$ and $T$ ( $U$ and $V$, respectively) by deleting all occurrences of formulas of the form 
$A(\bar{A}$, respectively), if there are any. Thus, in the case of the resolution rule, sequences of formulas are forced to "behave" like ordinary sets.

Let us stress that we do not restrict the applications of $\mathbf{R}_{r e s}$ to clauses, that is, not only formulas $A$ and $\bar{A}$, but also the elements of $S, T, U$ and $V$ may be compound. Thus, as we have mentioned before, $\mathbb{E}_{\text {res }}^{\mathrm{CPL}}$ constitutes an account of the non-clausal resolution.

Rewriting the premise sequents in the conclusion of rule $\mathbf{R}_{r e s}$ is to warrant a semantic invertibility of the rule (see the next Section). Another side-effect of rewriting the sequents is that in the case of a possible implementation of the method no backtracking would be needed in applying the resolution rule.

When the erotetic rules are applied to the initial question, one arrives at a sequence of questions called Socratic transformation. More formally,

Definition 5.1. (s-transformation). A finite sequence $\mathbf{s}=\left\langle Q_{1}, \ldots, Q_{n}\right\rangle$ is a Socratic transformation of the question $Q$ via the rules of $\mathbb{E}_{\text {res }}^{\mathrm{CP}}$ iff $Q_{1}=Q$ and $Q_{i+1}$ results from $Q_{i}(1 \leq i<n)$ by an application of a rule of $\mathbb{E}_{\text {res }}^{\mathrm{CP}}$.

For simplicity, Socratic transformations will be called s-transformations. Calculus $\mathbb{E}_{\text {res }}^{\mathrm{CP}}$ is a resolution system, thus we will need the notion of refutation in the system. This is given below.

Definition 5.2. (s-refutation). Let $\dashv A$ be a reversed sequent. A Socratic refutation of sequent $\dashv A$ in $\mathbb{E}_{\text {res }}^{\mathrm{CPL}}$ is a finite s-transformation $\mathbf{s}=$ $\left\langle Q_{1}, \ldots, Q_{n}\right\rangle$ of the question $Q_{1}=$ ? $(\dashv A)$ via the rules of $\mathbb{E}_{\text {res }}^{\mathrm{CPL}}$, such that the last question, $Q_{n}$, of $\mathbf{s}$ has the following form:

(\#) $\quad ?(\dashv \emptyset ; \Omega)$

where $\Omega$ is a finite sequence of sequents.

Socratic refutations will be called s-refutations, for short. If there is an s-refutation of a sequent, $\dashv A$, in $\mathbb{E}_{\text {res }}^{\mathrm{CPL}}$, then we say that $\dashv A$ is refutable in $\mathbb{E}_{\text {res }}^{\mathrm{CP}}$. Observe that we refute reversed sequents not the formula concerned by the sequent. Thus, as we shall see in a moment (see Theorem 5.5), an srefutation of $\dashv A$ proves $A$, just as a refutation of $\neg A$ in a standard resolution system proves $A$.

An s-transformation $\mathbf{s}$ is said to be eliminative iff all the rules which were used in constructing it are eliminative, i.e. rule $\mathbf{R}_{r e s}$ has not been applied. A resolution s-transformation ( $r$-transformation for short) is an s-transformation that was constructed be means of $\mathbf{R}_{r e s}$ only.

Definition 5.3. (s-extension). Let $\mathbf{s}=\left\langle Q_{1}, \ldots, Q_{n}\right\rangle$ and $\mathbf{s} *=\left\langle Q_{1}^{*}, \ldots, Q_{n}^{*}\right\rangle$ be Socratic transformations in $\mathbb{E}_{\text {res }}^{\mathrm{CP}}$. We say that $\mathbf{s}$ s-extends $\mathbf{s} *$ iff $Q_{1}$ results 
from $Q_{n}^{*}$ by an application of an erotetic rule of $\mathbb{E}_{\text {res }}^{\mathrm{CPL}}$. Then $\mathbf{s}$ is called an s-extension of $\mathbf{s} *$.

DeFinition 5.4. (complete s-transformation). An s-transformation $\mathbf{s}$ is said to be complete iff there is no s-extension of $\mathbf{s}$. An s-transformation $\mathbf{s}$ is said to be e-complete iff there is no eliminative s-extension of $\mathbf{s}$.

Let us consider, as an example, the following s-transformation, which we will call t:

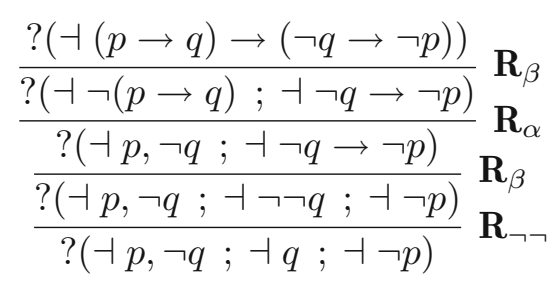

This s-transformation is eliminative and e-complete but it is not complete for we can s-extend it by the transformation $\mathbf{t}^{*}$ :

$$
\frac{\frac{\ldots}{?(\dashv p ; \dashv \neg p ; \dashv p, \neg q ; \dashv q)} \mathbf{R}_{r e s}}{?(\dashv \emptyset ; \dashv p, \neg q ; \dashv q ; \dashv p ; \dashv \neg p)} \mathbf{R}_{r e s}
$$

Recall that under our intended interpretation of sequents in terms of refutability the answer to a question containing ' $\dashv \emptyset$ ' as its constituent must be negative, for one cannot refute any element of the empty disjunction. Observe also that the concatenation of s-transformations $\mathbf{t}$ and $\mathbf{t}^{*}$ is an srefutation of the sequent $\dashv(p \rightarrow q) \rightarrow(\neg q \rightarrow \neg p)$.

Now we will prove that the rules of $\mathbb{E}_{\text {res }}^{\mathrm{CPL}}$ preserve some approppriate semantic properties. Namely, we say that a given rule preserves (in)correctness in $\mathrm{L}$ iff the fact that the premise sequence is (in)correct under an $\mathrm{L}$ valuation $v$ implies that the conclusion sequence is (in)correct under $v$. Now we show that the rules of $\mathbb{E}_{\text {res }}^{\mathrm{CP}}$ preserve both correctness and incorrectness in CPL.

LEMMA 1. Each rule of $\mathbb{E}_{\text {res }}^{\mathrm{CPL}}$ preserves incorrectness in $\mathrm{CPL}$.

Proof. Let us consider $\mathbf{R}_{\alpha}$ as an example. Let $v$ be an arbitrary CPLvaluation. Assume that the premise sequence $\mathbf{p}=\Phi ; \dashv S^{\prime} \alpha^{\prime} T ; \Psi$ is incorrect under $v$. Then at least one sequent from $\mathbf{p}$ is incorrect under $v$. The only non-trivial case is that when the sequent $\dashv S^{\prime} \alpha^{\prime} T$ is incorrect under $v$. Then it follows that $v(\alpha)=1$ and, naturally, also $v\left(\alpha_{1}\right)=v\left(\alpha_{2}\right)=1$. Consequently, the sequent $\dashv S^{\prime} \alpha_{1}{ }^{\prime} \alpha_{2}{ }^{\prime} T$ is incorrect under $v$, and hence the sequence $\mathbf{p}^{*}=\Phi ; \dashv S^{\prime} \alpha_{1}{ }^{\prime} \alpha_{2}{ }^{\prime} T ; \Psi$ is incorrect under $v$ as well. 
Lemma 2. Each rule of $\mathbb{E}_{\text {res }}^{\mathrm{CPL}}$ preserves correctness in $\mathrm{CPL}$.

Proof. Let us consider rule $\mathbf{R}_{\alpha}$ again. Let $w$ be an arbitrary CPL-valuation and let $\mathbf{q}=\Phi ; \dashv S^{\prime} \alpha_{1}{ }^{\prime} \alpha_{2}{ }^{\prime} T$; $\Psi$ be incorrect under $w$. We will deal only with the non-trivial case: the sequent $\dashv S^{\prime} \alpha_{1}{ }^{\prime} \alpha_{2}{ }^{\prime} T$ is incorrect under $w$. Naturally, $w\left(\alpha_{1}\right)=w\left(\alpha_{2}\right)=1$. Consequently, $w(\alpha)=1$ and thus both: sequent $\dashv S^{\prime} \alpha^{\prime} T$, and sequence $\mathbf{q}^{*}=\Phi ; \dashv S^{\prime} \alpha^{\prime} T ; \Psi$ are incorrect under $w$.

Observe that Lemma 2 actually proves the invertibility of the rules of $\mathbb{E}_{\text {res }}^{\mathrm{CPL}}$ - that is, the fact that they transmit incorrectness from the conclusion sequence to the premise sequence. ${ }^{7}$

Finally, let us prove that $\mathbb{E}_{\text {res }}^{\mathrm{CPL}}$ is sound with respect to the semantics of CPL.

TheOREM 5.5. (soundness). If sequent $\dashv A$ is refutable in $\mathbb{E}_{\text {res }}^{\mathrm{CP}}$, then the formula $A$ is CPL-valid.

Proof. From Corollary 4.4, Lemma 2 and Corollary 4.2.

\subsection{Erotetic Calculus for CLuN}

To formulate a complete set of rules characterizing the logic CLuN we need to enrich the language $\mathcal{L}_{\mathrm{CLuN}}$ with the following operator: $\chi$. We will denote the resulting language by the symbol $\mathcal{L}_{\mathrm{CLuN}^{+}}$. The set of formulas of $\mathcal{L}_{\mathrm{CLuN}}{ }^{+}$ is defined as the smallest set such that: (i) each formula of $\mathcal{L}_{\mathrm{CLuN}}$ is a formula of $\mathcal{L}_{\mathrm{CLuN}^{+}}$; (ii) if ' $\sim A^{\prime}$ ' is a formula of $\mathcal{L}_{\mathrm{CLuN}}$, then ' $\chi \sim A$ ' and ' $\neg \chi \sim A$ ' are formulas of $\mathcal{L}_{\mathrm{CLuN}^{+}}$.

Following [30] we use the additional operator $\chi$ in order to express the fact that paraconsistently negated formulas of $\mathcal{L}_{\mathrm{CLuN}}$ can get the direct assignment of a logical value.

The notion of CLuN-valuation for the language $\mathcal{L}_{\mathrm{CLuN}^{+}}$is obtained from the definition of CLuN-valuation for the language $\mathcal{L}_{\mathrm{CLuN}}$ by the addition of the following two clauses:

$$
\begin{gathered}
(\chi) \sigma(\chi \sim A)=1 \text { iff } \bar{\sigma}(\sim A)=1 \\
(\neg \chi) \sigma(\neg \chi \sim A)=1 \text { iff } \bar{\sigma}(\sim A)=0
\end{gathered}
$$

Taking language $\mathcal{L}_{\mathrm{CLuN}^{+}}$as $\mathcal{L}_{\mathrm{L}}$ we define the appropriate syntactic notions according to the pattern presented in Sect. 2. Erotetic calculus for the logic CLuN may be obtained from the calculus $\mathbb{E}_{\text {res }}^{\mathrm{CP}}$ by adding the following two

\footnotetext{
${ }^{7}$ We refer to the semantic notion of the invertibility of the rules here; for a syntactic version see [18, p. 19].
} 
rules for CLuN-negation. The resulting calculus will be denoted by the symbol $\mathbb{E}_{\text {res }}^{\mathrm{CLuN}}$.

$$
\begin{gathered}
\frac{?\left(\Phi ; \dashv S^{\prime} \sim A^{\prime} T ; \Psi\right)}{?\left(\Phi ; \dashv S^{\prime} \neg A^{\prime} T ; \dashv S^{\prime} \chi \sim A^{\prime} T ; \Psi\right)} \mathbf{R}_{\sim}^{\mathrm{CLuN}} \\
\frac{?\left(\Phi ; \dashv S^{\prime} \neg \sim A^{\prime} T ; \Psi\right)}{?\left(\Phi ; \dashv S^{\prime} A^{\prime} \neg \chi \sim A^{\prime} T ; \Psi\right)} \mathbf{R}_{\neg \sim}^{\mathrm{CLuN}}
\end{gathered}
$$

Lemma 3. Each rule of $\mathbb{E}_{\text {res }}^{\mathrm{CLuN}}$ preserves incorrectness in CLuN.

Proof. We will show only that the lemma holds for $\mathbf{R}_{\sim}^{\text {CLuN }}$. In the case of the other rules the argument will be analogous. Let $\sigma$ be an arbitrary CLuNvaluation and let the sequence ' $\Phi$; $\dashv S^{\prime} \sim A^{\prime} T$; $\Psi$ ' be incorrect under $\sigma$. Assume the non-trivial scenario: sequent $\dashv S^{\prime} \sim A^{\prime} T$ is incorrect under $\sigma$. Then, obviously, formula $\sim A$ is true under $\sigma$. According to Definition 3.2 of CLuN-valuation and to clause $(\chi)$ above, $\sigma(\neg A)=1$ or $\sigma(\chi \sim A)=1$. It follows that sequent $\dashv S^{\prime} \neg A^{\prime} T$ is incorrect under $\sigma$ or sequent $\dashv S^{\prime} \chi \sim A^{\prime} T$ is incorrect under $\sigma$. Consequently, sequence ' $\Phi$; $\dashv S^{\prime} \neg A^{\prime} T ; \dashv S^{\prime} \chi \sim A^{\prime} T$; $\Psi$ ' is incorrect under $\sigma$.

LEMma 4. (invertibility). Each rule of $\mathbb{E}_{\text {res }}^{\mathrm{CLuN}}$ is invertible, that is, preserves correctness in CLuN.

Proof. As an example let us analyse the rule $\mathbf{R}_{\neg \sim}^{\mathrm{CLuN}}$. Let $\sigma$ be an arbitrary CLuN-valuation and let ' $\Phi$; $\dashv S^{\prime} A^{\prime} \neg \chi \sim A^{\prime} T$; $\Psi$ ' be incorrect under $\sigma$. Then by definition 3.2 and clause $(\neg \chi)$ above (the non-trivial scenario), $\sigma(A)=1$ and $\sigma(\neg \chi \sim A)=1$. Then of course both: sequent $\dashv S^{\prime} \neg \sim A^{\prime} T$ and sequence ' $\Phi$; $\dashv S^{\prime} \neg \sim A^{\prime} T$; $\Psi^{\prime}$ are incorrect under $\sigma$.

The notions of s-transformation and s-refutation are defined as in the classical case. In fact, in the case of $L=C L u N s$ and $L=m b C$ these notions will be also defined according to Definition 5.1 and 5.2.

THeOREM 5.6. (soundness). Let $A$ be a formula of language $\mathcal{L}_{\mathrm{CLuN}}$. If sequent $\dashv A$ is refutable in $\mathbb{E}_{\text {res }}^{\mathrm{CLuN}}$, then $A$ is CLuN-valid.

Proof. Observe that if $A$ is a formula of $\mathcal{L}_{\mathrm{CLuN}}$, then it is also a formula of $\mathcal{L}_{\text {CLuN }}^{+}$and then, quite trivially, $A$ is true under every CLuN-valuation for the language $\mathcal{L}_{\text {CLuN }}$ iff $A$ is true under every CLuN-valuation for the language $\mathcal{L}_{\text {CLuN }}^{+}$. The theorem follows from this observation, Corollary 4.4, Lemma 4 and Corollary 4.2 . 


\subsection{Erotetic Calculus for CLuNs}

Let $\mathcal{L}_{\mathrm{CLuNs}^{+}}$be the language obtained from the language $\mathcal{L}_{\mathrm{CLuNs}}$ by the addition of the operator $\chi$ to $\mathcal{L}_{\mathrm{CLuNs}}$. The set of formulas of this language is defined in the same way as in the case of $\mathcal{L}_{\mathrm{CLuN}^{+}}$, but with the following restriction: ' $\chi \sim A$ ' and ' $\neg \chi \sim A$ ' are formulas of $\mathcal{L}_{\mathrm{CLuNs}^{+}}$iff formula $A$ is a propositional variable or $A=\neg B$ for some formula $B$ of $\mathcal{L}_{\mathrm{CLuNs}}$. Also the notion of CLuNs-valuation is extended to the formulas of language $\mathcal{L}_{\mathrm{CLuNs}^{+}}$ in much the same way as in the case of CLuN.

We will refer to the notation presented in Table 5.

Table 5. Extended $\alpha / \beta$ notation

\begin{tabular}{cccccc}
\hline$* \alpha$ & $* \alpha_{1}$ & $* \alpha_{2}$ & $* \beta$ & $* \beta_{1}$ & $* \beta_{2}$ \\
\hline$\neg \sim(A \wedge B)$ & $\neg \sim A$ & $\neg \sim B$ & $\sim(A \wedge B)$ & $\sim A$ & $\sim B$ \\
$\sim(A \vee B)$ & $\sim A$ & $\sim B$ & $\neg \sim(A \vee B)$ & $\neg \sim A$ & $\neg \sim B$ \\
$\sim(A \rightarrow B)$ & $A$ & $\sim B$ & $\neg \sim(A \rightarrow B)$ & $\neg A$ & $\neg \sim B$ \\
\hline
\end{tabular}

We define $\mathcal{L}_{-\mathrm{CLuNs}}{ }^{+}$as before. In order to obtain an erotetic calculus for CLuNs we add the following six rules to $\mathbb{E}_{\text {res }}^{\mathrm{CPL}}$ :

$$
\begin{gathered}
\frac{?\left(\Phi ; \dashv S^{\prime} * \alpha^{\prime} T ; \Psi\right)}{?\left(\Phi ; \dashv S^{\prime} * \alpha_{1}^{\prime} * \alpha_{2}{ }^{\prime} T ; \Psi\right)} \mathbf{R}_{* \alpha} \\
\frac{?\left(\Phi ; \dashv S^{\prime} * \beta^{\prime} T ; \Psi\right)}{?\left(\Phi ; \dashv S^{\prime} * \beta_{1}^{\prime} T ; \dashv S^{\prime} * \beta_{2}{ }^{\prime} T ; \Psi\right)} \mathbf{R}_{* \beta} \\
\frac{?\left(\Phi ; \dashv S^{\prime} \sim \sim A^{\prime} T ; \Psi\right)}{?\left(\Phi ; \dashv S^{\prime} A^{\prime} T ; \Psi\right)} \mathbf{R}_{\sim \sim 1} \\
\frac{?\left(\Phi ; \dashv S^{\prime} \neg \sim \sim A^{\prime} T ; \Psi\right)}{?\left(\Phi ; \dashv S^{\prime} \neg A^{\prime} T ; \Psi\right)} \mathbf{R}_{\sim 2} \\
\frac{?\left(\Phi ; \dashv S^{\prime} \sim A^{\prime} T ; \Psi\right)}{?\left(\Phi ; \dashv S^{\prime} \neg A^{\prime} T ; \dashv S^{\prime} \chi \sim A^{\prime} T ; \Psi\right)} \mathbf{R}_{\sim}^{\mathrm{CLuNs}} \\
\frac{?\left(\Phi ; \dashv S^{\prime} \neg \sim A^{\prime} T ; \Psi\right)}{?\left(\Phi ; \dashv S^{\prime} A^{\prime} \neg \chi \sim A^{\prime} T ; \Psi\right)} \mathbf{R}_{\neg \sim}^{\mathrm{CLuNs}}
\end{gathered}
$$

Rules $\mathbf{R}_{\sim}^{\mathrm{CLuNs}}$ and $\mathbf{R}_{\neg \sim}^{\mathrm{CLuNs}}$ may be applied, provided that $A \in \mathrm{VAR}$ or $A$ is of the form ' $\neg B$ '.

The resulting calculus is named $\mathbb{E}_{\text {res }}^{\mathrm{CLuNs}}$. The notions of s-transformation and s-refutation are understood as before. The proofs of the following lemmas are easy to produce and we will skip them here.

LEMMA 5. Each rule of $\mathbb{E}_{\text {res }}^{\text {CLuNs }}$ preserves incorrectness in CLuNs. 
Lemma 6. (invertibility). Each rule of $\mathbb{E}_{\text {res }}^{\mathrm{CLuNs}}$ is invertible, that is, preserves correctness in CLuNs.

Referring to invertibility of rules of $\mathbb{E}_{\text {res }}^{\text {CLuNs }}$ one may easily establish:

Theorem 5.7. (soundness). Let $A$ be a formula of language $\mathcal{L}_{\text {CLuns. If }}$ sequent $\dashv A$ is refutable in $\mathbb{E}_{\text {res }}^{\text {CLuNs }}$, then $A$ is CLuNs-valid.

\subsection{Erotetic Calculus for $\mathrm{mbC}$}

Let $\mathcal{L}_{\mathrm{mbC}}+$ be the language obtained from the language $\mathcal{L}_{\mathrm{mbC}}$ by the addition of the operator $\chi$ to $\mathcal{L}_{\mathrm{mbc}}$. The set of formulas of this language is the smallest set such that: (i) each formula of $\mathcal{L}_{\mathrm{mbC}}$ is a formula of $\mathcal{L}_{\mathrm{mbC}}$; (ii) if $A$ is a formula of $\mathcal{L}_{\mathrm{mbC}}$, where $A=\sim B$ or $A=\circ B$, then ' $\chi A$ ' and ' $\neg \chi A$ ' are formulas of $\mathcal{L}_{\mathrm{mbC}^{+}}$.

The notion of $\mathrm{mbC}$-valuation for the language $\mathcal{L}_{\mathrm{mbC}}+$ is obtained from the definition of $\mathrm{mbC}$-valuation for the language $\mathcal{L}_{\mathrm{mbC}}$ by the addition of the following four clauses:

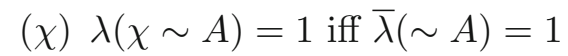

$(\neg \chi) \lambda(\neg \chi \sim A)=1$ iff $\bar{\lambda}(\sim A)=0$

$\left(\chi^{\prime}\right) \lambda(\chi \circ A)=1$ iff $\bar{\lambda}(\circ A)=1$

$\left(\neg \chi^{\prime}\right) \lambda(\neg \chi \circ A)=1$ iff $\bar{\lambda}(\circ A)=0$

We obtain the dual erotetic calculus $\mathbb{E}_{\text {res }}^{\mathrm{mbC}}$ for logic $\mathrm{mbC}$ by adding the following two rules for the consistency operator to $\mathbb{E}_{\text {res }}^{\mathrm{CLuN}}$ :

$$
\begin{gathered}
\frac{?\left(\Phi ; \dashv S^{\prime} \circ A^{\prime} T ; \Psi\right)}{?\left(\Phi ; \dashv S^{\prime} \neg A^{\prime} \chi \circ A^{\prime} T ; \dashv S^{\prime} \neg \sim A^{\prime} \chi \circ A^{\prime} T ; \Psi\right)} \mathbf{R}_{\circ} \\
\frac{?\left(\Phi ; \dashv S^{\prime} \neg \circ A^{\prime} T ; \Psi\right)}{?\left(\Phi ; \dashv S^{\prime} A^{\prime} \sim A^{\prime} T ; \dashv S^{\prime} \neg \chi \circ A^{\prime} T ; \Psi\right)} \mathbf{R}_{\neg \circ}
\end{gathered}
$$

LEMMA 7. Each rule of $\mathbb{E}_{\text {res }}^{\mathrm{mbC}}$ preserves incorrectness in $\mathrm{mbC}$.

Proof. Let us examine as an example the rule $\mathbf{R}_{\circ}$. Let $\lambda$ be an arbitrary $\mathrm{mbC}$-valuation. Assume that the sequent $\dashv S^{\prime} \circ A^{\prime} T$ is incorrect under $\lambda$ (the only non-trivial case). Then $\lambda(\circ A)=1$ and according to the definition of $\mathrm{mbC}$-valuation and the clause $\left(\chi^{\prime}\right)$ above: $\neg A$ and $\chi \circ A$ are both true under $\lambda$ or $\neg \sim A$ and $\chi \circ A$ are both true under $\lambda$. It means that either sequent $\dashv S^{\prime} \neg A^{\prime} \chi \circ A^{\prime} T$ is incorrect under $\lambda$ or sequent $\dashv S^{\prime} \neg \sim A^{\prime} \chi \circ A^{\prime} T$ is incorrect under $\lambda$.

Lemma 8. (invertibility). Each rule of $\mathbb{E}_{\text {res }}^{\mathrm{mbC}}$ is invertible, that is, preserves correctness in $\mathrm{mbC}$. 
Proof. We will consider rule $\mathbf{R}_{\circ}$ again. Let $\lambda$ be an arbitrary $\mathrm{mbC}$-valuation. Assume that at least one of the sequents $\dashv S^{\prime} \neg A^{\prime} \chi \circ A^{\prime} T, \dashv S^{\prime} \neg \sim$ $A^{\prime} \chi \circ A^{\prime} T$ is incorrect under $\lambda$. In either case $\lambda(\chi \circ A)=1$, and from the clause $\left(\chi^{\prime}\right)$ above it follows that $\bar{\lambda}(\circ A)=1$. Moreover, either $\lambda(A)=$ 0 or $\lambda(\sim A)=0$. Consequently, the sequent $\dashv S^{\prime} \circ A^{\prime} T$ is incorrect under $\lambda$.

As in the previous cases, invertibility of the rules allows one to establish soundness.

THEOREM 5.8. (soundness). Let $A$ be a formula of language $\mathcal{L}_{\mathrm{mbC}}$. If sequent $\dashv A$ is refutable in $\mathbb{E}_{\text {res }}^{\mathrm{mbC}}$, then $A$ is $\mathrm{mbC}$-valid.

\section{Examples of s-proofs}

s-refutation in $\mathbb{E}_{\text {res }}^{\text {CLuN }}$. Formula $(p \vee \sim p)$ is CLuN-valid.

$$
\frac{\frac{?(\dashv p \vee \sim p)}{?(\dashv p ; \dashv \sim p)} \mathbf{R}_{\beta}}{\frac{?(\dashv p ; \dashv \neg p ; \dashv \chi \sim p)}{?(\dashv \emptyset ; \dashv \chi \sim p ; \dashv p ; \dashv \neg p)} \mathbf{R}_{\sim}} \mathbf{R}_{r e s}
$$

s-refutation in $\mathbb{E}_{\text {res }}^{\text {CLuNs }}$. Formula $p \rightarrow \sim \sim p$ is CLuNs-valid.

$$
\begin{aligned}
& \frac{?(\dashv p \rightarrow \sim \sim p)}{?(\dashv \neg p ; \dashv \sim \sim p)} \mathbf{R}_{\beta} \\
& \frac{?(\dashv \neg p ; \dashv p)}{?(\dashv \emptyset ; \dashv \neg p ; \dashv p)} \mathbf{R}_{\sim \sim 1} \\
& \mathbf{R}_{r e s}
\end{aligned}
$$

s-refutations in $\mathbb{E}_{\text {res }}^{\mathrm{mbC}}$. Formula $(p \rightarrow q) \wedge \circ q \rightarrow(\sim q \rightarrow \sim p)$ is mbC-valid.

$$
\begin{gathered}
\frac{?(\dashv(p \rightarrow q) \wedge \circ q \rightarrow(\sim q \rightarrow \sim p))}{?(\dashv \neg((p \rightarrow q) \wedge \circ q) ; \dashv \sim q \rightarrow \sim p)} \mathbf{R}_{\beta} \\
\frac{? ?(\dashv \neg(p \rightarrow q) ; \dashv \neg \circ q ; \dashv \sim q \rightarrow \sim p)}{\frac{?(\dashv \neg(p \rightarrow q) ; \dashv \neg \circ q ; \dashv \neg \sim q ; \dashv \sim p)}{?_{\alpha}}} \mathbf{R}_{\beta} \\
\frac{\frac{?(\dashv p, \neg q ; \dashv \neg \circ q ; \dashv \neg \sim q ; \dashv \sim p)}{?(\dashv p, \neg q ; \dashv q, \sim q ; \dashv \neg \chi \circ q ; \dashv \neg \sim q ; \dashv \sim p)} \mathbf{R}_{\neg \circ}}{\frac{?(\dashv p, \neg q ; \dashv q, \sim q ; \dashv \neg \chi \circ q ; \dashv \neg \sim q ; \dashv \neg p ; \dashv \chi \sim p)}{\sim} \mathbf{R}_{\sim}} \\
\frac{\frac{?(\dashv \neg q ; \dashv q, \sim q ; \dashv \neg \chi \circ q ; \dashv \neg \sim q ; \dashv \chi \sim p ; \dashv p, \neg q ; \dashv \neg p)}{? ?(\dashv \sim q ; \dashv \neg \chi \circ q ; \dashv \neg \sim q ; \dashv \chi \sim p ; \dashv p, \neg q ; \dashv \neg p ; \dashv \neg q ; \dashv q, \sim q)} \mathbf{R}_{r e s}}{?(\dashv \emptyset ; \dashv \neg \chi \circ q ; \dashv \chi \sim p ; \dashv p, \neg q ; \dashv \neg p ; \dashv \neg q ; \dashv q, \sim q ; \dashv \sim q ; \dashv \neg \sim q)} \mathbf{R}_{r e s}
\end{gathered}
$$




\section{Completeness}

There are two central concepts which we will use in the completeness proof: that of complete and minimal resolution transformation (see below) and that of a semantic tree. Semantic trees have been used in the proof of completeness of resolution for CPL in [20]. We rely on the version presented in [10], but adjust it to the dual format of the rules. We extend the method of proving completeness with semantic trees to the non-classical cases.

\subsection{Resolution-Transformations}

Recall that an $r$-transformation is a Socratic transformation which was constructed by means of $\mathbf{R}_{\text {res }}$ only. We will say that an r-transformation is complete and minimal, or that it is a cmr-transformation iff (i) either it is finite and the last question contains, int.al., the empty sequent, or else $\mathbf{R}_{\text {res }}$ is applied to all pairs of sequents to which it can be applied (completeness), and (ii) $\mathbf{R}_{r e s}$ is never applied more than once to a given pair of sequents with respect to the same formula (minimality).

The notion of cmr-transformation is pivotal for the proof of completeness of each dual erotetic calculus we are considering here. We make this concept sufficiently exact by constructing an algorithm that generates for a given question a cmr-transformation of that question. It is easy to observe that this algorithm terminates, i.e. each cmr-transformation of an arbitrary question is finite.

Let us first introduce the notion of memory sequence of a question in s-transformation $\mathbf{s}\left(\mathrm{m}_{\mathbf{s}}\right.$-sequence for short).

Definition 7.1. (memory sequence). Let $\mathbf{s}=\left\langle Q_{1}, \ldots, Q_{n}\right\rangle$ be an s-transformation of question $Q=Q_{1}$ via the rules of $\mathbb{E}_{\text {res }}^{\mathrm{L}}$. An $\mathrm{m}_{\mathbf{s}}$-sequence of the question $Q_{n}$ in s-transformation $\mathbf{s}$ (formally: $\mathrm{m}_{\mathbf{s}} Q_{n}$ ) is a (possibly empty) sequence of sets of sequents which satisfies the following condition:

( $\star$ ) a set $\left\{\dashv S^{\prime} A^{\prime} T^{[\overline{\bar{A}}]}, \dashv U^{\prime} \bar{A}^{\prime} V^{[\overline{\bar{A}}]}\right\}$ is a term of $\mathrm{m}_{\mathbf{s}} Q_{n}$ iff there exists question $Q_{i}$ of the form:

(i) $Q_{i}=?\left(\Phi ; \dashv S^{\prime} A^{\prime} T ; \Psi ; \dashv U^{\prime} \bar{A}^{\prime} V ; \Omega\right)$ where $1 \leq i<n$, such that question $Q_{i+1}$ of the following form

(i) $Q_{i+1}=?\left(\dashv \underline{S}^{\prime} \underline{T}^{\prime} \underline{U}^{\prime} \underline{V} ; \Phi ; \Psi ; \Omega ; \dashv S^{\prime} A^{\prime} T ; \dashv U^{\prime} \bar{A}^{\prime} V\right)$ results from $Q_{i}$ by an application of the rule $\mathbf{R}_{\text {res }}$ to sequents $\dashv S^{\prime} A^{\prime} T$ and $\dashv U^{\prime} \bar{A}^{\prime} V$ with respect to formula $\overline{\bar{A}}$.

Let us note that there are questions such that some of their r-transformations are infinite. However, such r-transformations are contaminated 
with severe redundancy: they appear when we apply the resolution rule repeatedly to the same pair of sequents with respect to the same formula. A method of deriving non-redundant complete r-transformations (cmr-transformations) is defined in Algorithm 1.

Input: A question $Q$ of $\mathcal{L}_{\dashv L}^{?}$ based on sequence $\Phi$, which consists of $m$ terms Output: A finite cmr-transformation of $Q$ begin

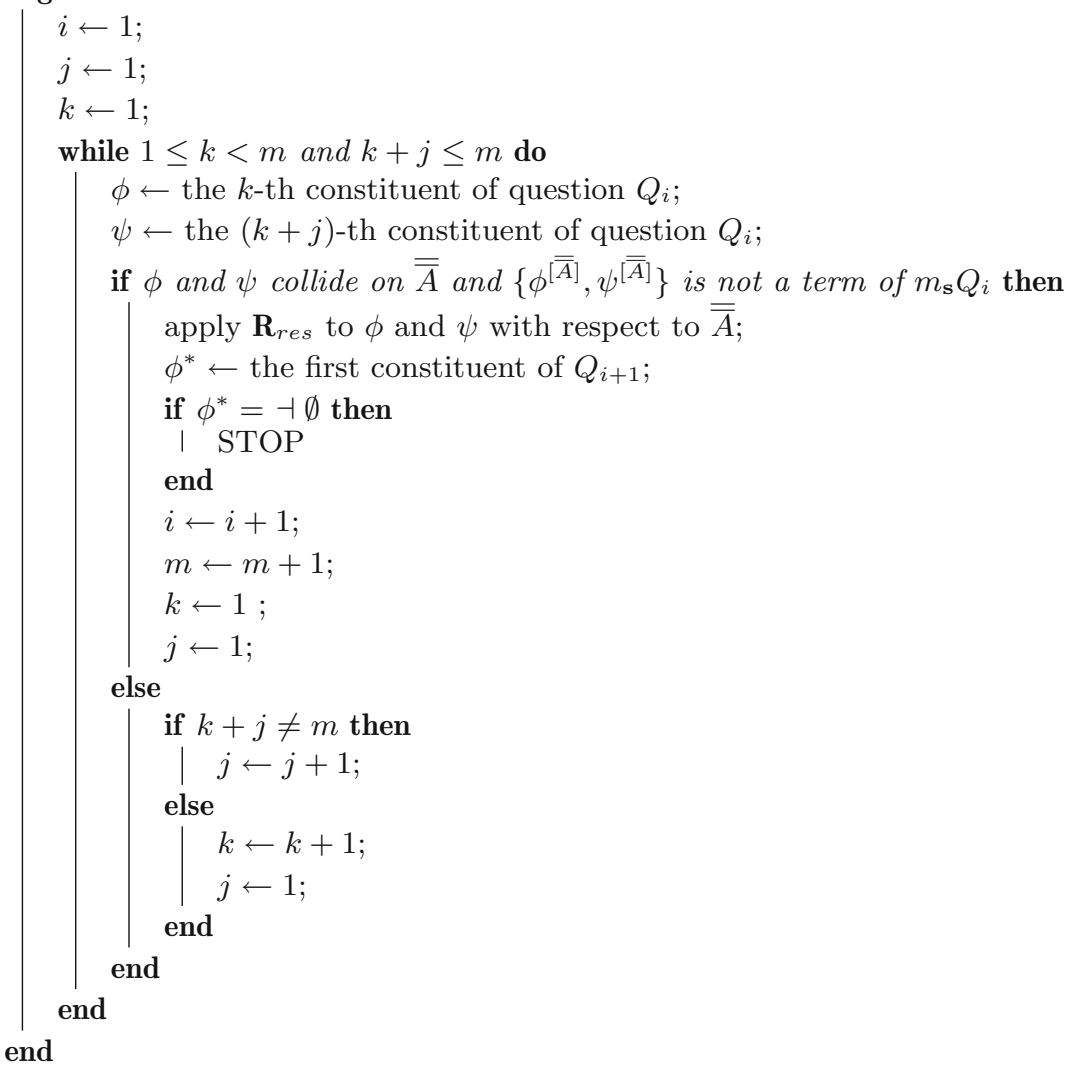

Algorithm 1: cmr-transformation of a question of $\mathcal{L}_{-\mathrm{L}}^{?}$

Let us consider the following example:

$$
\begin{aligned}
& Q_{1}=?(\dashv p, q, \neg r ; \dashv \neg q ; \dashv \neg p ; \dashv r) \\
& Q_{2}=?(\dashv p, \neg r ; \dashv \neg p ; \dashv r ; \dashv p, q, \neg r ; \dashv \neg q) \\
& Q_{3}=?(\dashv \neg r ; \dashv r ; \dashv p, q, \neg r ; \dashv \neg q ; \dashv p, \neg r ; \dashv \neg p) \\
& Q_{4}=?(\dashv \emptyset ; \dashv p, q, \neg r ; \dashv \neg q ; \dashv p, \neg r ; \dashv \neg p ; \dashv \neg r ; \dashv r)
\end{aligned}
$$


The above cmr-transformation stops due to the appearance of $\dashv \emptyset$. The memory sequence of question $Q_{4}$ is the following:

$$
\mathrm{m}_{\mathrm{s}} Q_{4}=\left\langle\left\{\dashv p, q, \neg r^{[q]}, \dashv \neg q^{[q]}\right\},\left\{\dashv p, \neg r^{[p]}, \dashv \neg p^{[p]}\right\},\left\{\dashv \neg r^{[r]}, \dashv r^{[r]}\right\}\right\rangle
$$

Theorem 7.2. (termination). For each question $Q$ of $\mathcal{L}_{-\mathrm{LL}}^{\text {? }}$, each cmr-transformation of question $Q$ is finite, ${ }^{8}$ i.e. Algorithm 1 does terminate.

Proof. To put it in terms of resolution, the idea of the proof is that questions of $\mathcal{L}_{-J L}^{?}$ are always based on finite sequences of sequents and each sequent is based on a finite sequence of formulas, hence there is always a finite number of "clauses" to be used, and the resolution rule $\left(\mathbf{R}_{r e s}\right)$ is never applied twice in the same manner.

\subsection{Semantic Trees}

Recall that $\mathrm{L}$ stands for CPL, CLuN, CLuNs or mbC.

Definition 7.3. (L-unanalysable formulas).

1. All literals, and only literals, are CPL-unanalysable formulas. Variables will be called positive CPL-unanalysable formulas.

2. The formulas of one of the following forms: (i) $p_{i}$; (ii) $\neg p_{i}$; (iii) $\chi \sim A$; (iv) $\neg \chi \sim A$, and only them, are CLuN-unanalysable formulas. Formulas of the form: (i) and (iii) are called positive CLuN-unanalysable formulas.

3. The formulas of one of the following forms: (i) $p_{i}$; (ii) $\neg p_{i}$; (iii) $\chi \sim p_{i}$; (iv) $\neg \chi \sim \neg A$, and only them, are CLuNs-unanalysable. Formulas of the form (i) and (iii) are called positive CLuNs-unanalysable formulas.

4. The formulas of one of the following forms: (i) $p_{i}$; (ii) $\neg p_{i}$; (iii) $\chi \sim A$; (iv) $\neg \chi \sim A ;(\mathrm{v}) \chi \circ A$; (vi) $\neg \chi \circ A$, and only them, are mbC-unanalysable. Formulas of the form (i), (iii) and (v) are called positive mbC-unanalysable formulas.

For each set $V$ of L-unanalysable formulas we define the set $V_{P}$ of positive L-unanalysable formulas based on the set $V$ : (i) every positive L-unanalysable formula from $V$ belongs to $V_{P}$, (ii) if $\neg A \in V$, then $A \in V_{P}$, (iii) nothing else belongs to $V_{P}$.

Let us start with the following observation:

${ }^{8}$ Algorithm 1 is not deterministic, since the "resolution formula" $\overline{\bar{A}}$ in the body of the while loop is not clearly indicated. Therefore we assume that question $Q$ can have more than one cmr-transformation. (Obviously, the algorithm can be reformulated to be deterministic, but it was not necessary for our goals.) 


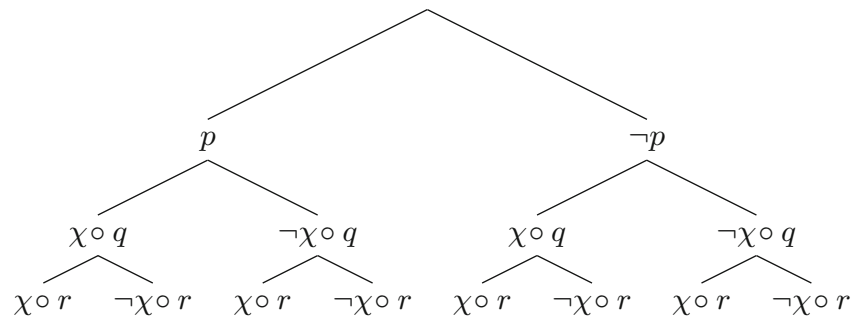

Figure 1. Semantic tree for the set $V=\{p, \neg \chi \circ q, \chi \circ r\}$ of $\mathrm{mbC}$ unanalysable formulas

Lemma 9. (simplification for $\mathrm{L})$. Let $Q=?(\dashv A)$ be a question of $\mathcal{L}_{-\mathrm{L}}^{\text {? }}$. There is an e-complete s-transformation $\mathbf{s}=\left\langle Q_{1}, \ldots, Q_{n}\right\rangle$ of the question $Q=Q_{1}$ such that only L-unanalysable formulas occur in the constituents of $Q_{n}$.

Proof. By induction on the complexity of $A$ for a given logic $\mathrm{L}$.

The concept of the level of a tree (or more precisely: the level of a node of a tree) is defined inductively as follows: (i) the level of the root node is 0 ; (ii) if the level of node $\mathrm{n}$ is $j$, then the level of each child of node $\mathrm{n}$ is $j+1$.

The length of a branch of a tree is the number of the nodes of that branch minus 1 . The depth of a tree $\mathrm{T}$ (symbolically $|\mathrm{T}|$ ) equals the length of the maximal branch of that tree.

Definition 7.4. (L-semantic tree). Let $V$ be the set of L-unanalysable formulas and let $V_{P}=\left\{B_{1}, \ldots, B_{k}\right\}$ be the set of positive L-unanalysable formulas based on $V$. An L-semantic tree $\mathrm{T}$ for set $V$ is a downward branching binary tree (where $|\mathrm{T}|=k$ ) with single root, such that the root has no label and for each node $\mathrm{n}$ on the level $j-1$ (where $1 \leq j \leq k$ ): the left child of $\mathrm{n}$ is labelled with $B_{j}$ and the right child of $\mathrm{n}$ is labelled with $\neg B_{j}$. Figure 1 presents an example of a semantic tree for a set of $\mathrm{mbC}$-unanalysable formulas.

\subsection{Completeness Proof for $\mathbb{E}_{\text {res }}^{\mathrm{CPL}}$}

For the purpose of the completeness proof we will use the symbol $\mathbf{C} Q$ to denote the set of constituents of the question $Q$. We will also say that set $\mathbf{C} Q$, where $Q=?(\Phi)$, is unsatisfiable in $\mathrm{L}$ iff the sequence $\Phi$ is incorrect under every L-valuation.

We may define a one-to-one correspondence between each branch of a semantic tree and a CPL-valuation as follows. If a literal $l$ labels a node of a branch $\mathrm{b}$, then $v_{\mathrm{b}}(l)=1$. For each propositional variable $p_{i}$ which does not 
occur on a tree, $v_{\mathrm{b}}\left(p_{i}\right)=0$. Observe that in the same way we may assign $\mathrm{L}$-valuations to branches of L-semantic trees for the other $\mathrm{L}$. This follows from the fact that logical values are assigned to the positive L-unanalysable formulas directly, i.e., independently of their structure.

Let $\mathrm{b}_{1}, \ldots, \mathrm{b}_{n}$ be all the branches of a tree $\mathrm{T}$ for a set of literals. We say that branch $\mathrm{b}_{i}$ (where $1 \leq i \leq n$ ) is compatible with a sequent $\phi$ iff whenever a CPL-unanalysable formula $F$ (i.e. a literal) occurs in sequent $\phi$, then $F$ labels some node of $\mathrm{b}_{i}$. For the definition in the case of the other $\mathrm{L}$ we simply put "L-unanalysable" instead of "CPL-unanalysable". Note that the trivial path from the root to itself is compatible only with the empty sequent $\dashv \emptyset$.

Theorem 7.5. (completeness for $\mathbb{E}_{\text {res }}^{\mathrm{CPL}}$ ). If a formula $A$ is CPL-valid, then the sequent $\dashv A$ is refutable in $\mathbb{E}_{\text {res }}^{\mathrm{CPL}}$.

Proof. (A modified version of the proof presented in [10]) Assume that $A$ is CPL-valid. We will show that in this case there exists an s-transformation s of the question $Q=?(\dashv A)$ such that the last question of $\mathbf{s}$ is based on (among others) the empty sequent $\dashv \emptyset$.

By Lemma 9 we know that there is an eliminative s-transformation $\mathbf{s}_{n}=$ $\left\langle Q_{1}, \ldots, Q_{i}\right\rangle$ of the question $Q_{1}=?(\dashv A)$, such that $Q_{i}$ is based on sequents containing literals only. Moreover, by Lemma 1 and 2 (and by induction), the set $\mathbf{C} Q_{1}$ is unsatisfiable in CPL iff the set $\mathbf{C} Q_{i}$ is unsatisfiable in CPL. Now if we assume that $A$ is CPL-valid, we have that $\mathbf{C} Q_{i}$ is unsatisfiable in CPL. Our aim is to prove that, assuming that $A$ is CPL-valid, by using $\mathbf{R}_{r e s}$ we can obtain a cmr-transformation $\mathbf{s}_{r e s}=\left\langle Q_{i}, \ldots, Q_{n}\right\rangle$ such that $\mathbf{C} Q_{n}$ contains the sequent $\dashv \emptyset$.

We construct a cmr-transformation $\mathbf{s}_{\text {res }}$ of question $Q_{i}$ according to Algorithm 1. From Theorem 7.2 it follows that $\mathbf{s}_{\text {res }}$ is finite. Moreover, the finite set $\mathbf{C}_{\mathbf{s}_{\text {res }}}$ of sequents of the sequence $\mathbf{s}_{\text {res }}$ is closed under $\mathbf{R}_{\text {res }}$ in the following sense. Assume that sequents $\phi \in \mathbf{C}_{\mathbf{s}_{r e s}}$ and $\psi \in \mathbf{C}_{\mathbf{s}_{r e s}}$ collide on a variable $p_{k}$. The sequent $\phi$ is a constituent of a question of $\mathbf{s}_{r e s}$, and similarly, the sequent $\psi$ is a constituent of a question of $\mathbf{s}_{\text {res }}$ (where the two questions may be different!). Only $\mathbf{R}_{r e s}$ is applied in the construction of $\mathbf{s}_{r e s}$ and the premise sequents are rewritten into the conclusion-question after each application of the rule. Therefore either the empty sequent is derived in $\mathbf{s}_{r e s}$, which is fine for us, or sequents $\phi$ and $\psi$ finally "meet" as constituents of one question. Then again, either the empty sequent occurs in $\mathbf{C}_{\mathbf{s}_{\text {res }}}$, or $\mathbf{R}_{\text {res }}$ is applied to some question of $\mathbf{s}_{r e s}$ with $\phi$ and $\psi$ as active and with respect to $p_{k}$, and then the "resolvent" (conclusion sequent) appears in $\mathbf{C}_{\mathbf{s}_{r e s}}$.

Let $\Delta$ be the set of all propositional variables which occur in sequents of $\mathbf{C} Q_{i}$. Consider a semantic tree $\mathrm{T}_{\Delta}$ for $\Delta$. We say that a branch $\mathrm{b}_{j}$ of $\mathrm{T}_{\Delta}$ is 
$\mathbf{C}_{\mathbf{s}_{\text {res }}}$-closed iff $\mathbf{b}_{j}$ is compatible with some sequent in $\mathbf{C}_{\mathbf{s}_{r e s}}$. We say a node $\mathrm{n}$ of $\mathrm{T}_{\Delta}$ is a success node if the path from the root of $\mathrm{T}_{\Delta}$ to $\mathrm{n}$ is $\mathbf{C}_{\mathbf{s}_{\text {res }}}$-closed.

Note that each branch of $\mathbf{T}_{\Delta}$ is $\mathbf{C}_{\mathbf{s}_{r e s}}$-closed. The rationale is the following: if there was a branch $\mathrm{b}_{i}$ which was not $\mathbf{C}_{\mathbf{s}_{r e s}}$-closed, then $\mathrm{b}_{i}$ would not be compatible with any element of $\mathbf{C}_{\mathbf{s}_{r e s}}$. But $\mathrm{T}_{\Delta}$ is a semantic tree for $\Delta$, thus for every sequent $\phi$ from $\mathbf{C}_{\mathbf{s}_{\text {res }}}$, and for each variable $p_{k}$ that occurs in $\phi$, branch $\mathrm{b}_{i}$ contains either $p_{k}$ or $\neg p_{k}$. Its non-compatibility with $\phi$ for every $\phi \in \mathbf{C}_{\mathbf{s}_{\text {res }}}$ amounts to the fact that each $\phi$ is correct under the valuation $v_{\mathrm{b}_{i}}$, and thus the set $\mathbf{C}_{\mathbf{s}_{\text {res }}}$ is correct under $v_{\mathrm{b}_{i}}$, contrary to our assumption that $\mathbf{C} Q_{i}$ is unsatisfiable in CPL.

Note that every branch of $\mathrm{T}_{\Delta}$ contains a success node. Let $* \mathrm{~T}_{\Delta}$ be a semantic tree obtained from $\mathrm{T}_{\Delta}$ by deleting every descendant of a success node in branches of $\mathrm{T}_{\Delta}\left(* \mathrm{~T}_{\Delta}\right.$ is a subtree of $\left.\mathrm{T}_{\Delta}\right)$. We are going to show that $* \mathrm{~T}_{\Delta}$ is the trivial tree, i.e a tree which contains just the root node. The consequence would be that the empty sequent $\dashv \emptyset$ belongs to the set $\mathbf{C}_{\mathbf{s}_{r e s}}$. Thus we assume that each branch of $* \mathrm{~T}_{\Delta}$ is $\mathbf{C}_{\mathbf{s}_{r e s}}$-closed and that $* \mathrm{~T}_{\Delta}$ is non-trivial, and we derive a contradiction.

Since $* \mathrm{~T}_{\Delta}$ is non-trivial, it contains a branch $\mathrm{m}$ of maximal length. Assume $\mathrm{m}$ is of the form m', $\mathrm{L}$ where $\mathrm{m}^{\prime}$ is the path from the root of $* \mathrm{~T}_{\Delta}$ to the parent of $\mathrm{L}$, and $\mathrm{L}$ is the left child (the case where $\mathrm{m}$ is of the form $\mathrm{m}^{\prime}, \mathrm{R}$ is analogous). It follows from the definition of $* \mathrm{~T}_{\Delta}$ that $\mathrm{L}$ is a success node, the branch $\mathrm{m}$ is $\mathbf{C}_{\mathbf{s}_{r e s}}$-closed and $\mathrm{m}$ ' is not $\mathbf{C}_{\mathbf{s}_{r e s}}$-closed. Let $\mathrm{R}$ be the right sibling of $\mathrm{L}$ in $\mathrm{T}_{\Delta}$. If $\mathrm{R}$ were not a success node, then the shortest $\mathbf{C}_{\mathbf{s}_{r e s}}$-closed path beginning with $\mathrm{m}^{\prime}, \mathrm{R}$ would be longer than $\mathrm{m}^{\prime}, \mathrm{L}$, which is of maximal length in $* \mathrm{~T}_{\Delta}$ from the assumption. So $R$ is a success node. Since $\mathrm{m}^{\prime}$ is not $\mathbf{C}_{\mathrm{s}_{r e s}}$-closed and each branch of $* \mathrm{~T}_{\Delta}$ is $\mathbf{C}_{\mathbf{s}_{\text {res }}}$-closed, it follows that $\mathrm{m}^{\prime}, \mathrm{R}$ is a branch of $* \mathrm{~T}_{\Delta}$.

Let $p_{k}$ label the node $\mathrm{L}$ and $\neg p_{k}$ label the node $\mathrm{R}$. The branches $\mathrm{m}^{\prime}, \mathrm{L}$ and $\mathrm{m}^{\prime}, \mathrm{R}$ are $\mathbf{C}_{\mathbf{s}_{\text {res }}}$-closed. It follows that there exists a sequent $\phi_{\mathrm{L}}$ from $\mathbf{C}_{\mathbf{s}_{\text {res }}}$ which is compatible with the branch $\mathrm{m}^{\prime}, \mathrm{L}$ and there exists a sequent $\phi_{\mathrm{R}}$ from $\mathbf{C}_{\mathbf{s}_{\text {res }}}$ which is compatible with the branch $\mathrm{m}^{\prime}, \mathbf{R}$. Since $\mathbf{C}_{\mathbf{s}_{\text {res }}}$ is closed under $\mathbf{R}_{\text {res }}$, we conclude that the empty sequent is in $\mathbf{C}_{\mathbf{s}_{\text {res }}}$ (which is fine) or $\mathbf{C}_{\mathbf{s}_{\text {res }}}$ contains a sequent, $\phi$, which is the conclusion sequent of a question obtained by an application of $\mathbf{R}_{r e s}$ to a question with $\phi_{\mathrm{L}}$ and $\phi_{\mathrm{R}}$ as active and with respect to $p_{k}$. It is easy to see that then the branch $\mathrm{m}^{\prime}$ is compatible with sequent $\phi$; and since $\phi \in \mathbf{C}_{\mathbf{s}_{r e s}}$, it follows that branch $\mathrm{m}^{\prime}$ is $\mathbf{C}_{\mathbf{s}_{r e s}}$-closed, so we arrive at a contradiction.

Consequently, $* \mathrm{~T}_{\Delta}$ is a trivial $\mathbf{C}_{\mathrm{s}_{\text {res }}}$-closed tree, such that the root of $* \mathrm{~T}_{\Delta}$ is a success node. Thus it follows that the empty sequent $\dashv \emptyset$ is an element of $\mathbf{C}_{\mathbf{s}_{r e s}}$. 
It is easy to see that the concatenation of eliminative s-transformations $\mathbf{s}_{n}=\left\langle Q_{1}, \ldots, Q_{i}\right\rangle$ of the question $Q_{1}=?(\dashv A)$ and its resolution s-extension $\mathbf{s}_{\text {res }}=\left\langle Q_{i+1}, \ldots, Q_{n}\right\rangle$ is an s-refutation of the sequent $\dashv A$.

\subsection{Completeness of $\mathbb{E}_{\text {res }}^{\mathrm{L}}$ for $\mathrm{L}=\mathrm{CLuN}, \mathrm{CLuNs}, \mathrm{mbC}$}

The following theorem is true:

THEOREM 7.6. (completeness for $\mathbb{E}_{\text {res }}^{\mathrm{L}}$ ). Let $A$ be a formula of language $\mathcal{L}_{\mathrm{L}}$. If $A$ is $\mathrm{L}$-valid, then the sequent $\dashv A$ is refutable in $\mathbb{E}_{\text {res }}^{\mathrm{L}}$.

Proof. The proof is analogous to the completeness proof for $\mathbb{E}_{\text {res }}^{\mathrm{CPL}}$. The main difference is due to the presence of $\chi$-formulas, but, as we remember, they get a direct assignment of a logical value, and thus semantically they behave much as propositional variables. From Lemma 9 we know that there exists a finite eliminative s-transformation of the question ? $(\dashv A)$ such that its last question contains only L-unanalysable formulas. We consider set $\Delta$ of all these formulas and an L-semantic tree $\mathrm{T}_{\Delta}$ for $\Delta$. What is essential in the concept of an L-semantic tree for $\Delta$ is that each path of such a tree corresponds to certain $\mathrm{L}$-valuation restricted to $\Delta$ and each $\mathrm{L}$-valuation restricted to some set $\Delta$ is represented by some path of an L-semantic tree for $\Delta$. This allows the argument to be constructed just as in the case of CPL: in order to obtain the version for $L$ it suffices to consider positive L-unanalysable formulas instead of propositional variables.

The reader may wonder to what extent the completeness result concerns the original account of CLuN, which was introduced without the classical negation $\neg$ (see [2]). Although erotetic calculi are worded in a richer language and thus directly concern formulas of certain lingustic extension of the original CLuN, their usage may be simply restricted to examine $\neg$-free formulas only. Obviously, in an s-refutation of $\dashv A$, where $A$ is $\neg$-free, the classical negation may still occur, but it serves proof-theoretical purposes only, and thus in the case of $\neg$-free formulas it could be replaced with the truth sign $\mathbf{F}$. To summarise this issue let us observe that by Theorems 5.6 and 7.6 we may establish the following result.

Theorem 7.7. Let $A$ be a $\neg$-free formula of $\mathcal{L}_{\mathrm{CLuN}}$. Then $A$ is CLuN-valid iff sequent $\dashv A$ is refutable in $\mathbb{E}_{\text {res }}^{\mathrm{CLuN}}$. 


\section{Erotetic Calculi and IEL}

For the sake of the size of this paper this section is very technical - we have only listed the definitions. For an introduction to Inferential Erotetic Logic see $[25,27]$.

As we have stressed before, erotetic calculi are primarly calculi of questions, and they can be interpreted from the viewpoint of Inferential Erotetic Logic. Here we present semantics for the languages $\mathcal{L}_{-\mathrm{H}}^{\text {? }}$ based on the notion of partition of a language. ${ }^{9}$ Then we define the notion of entailment in order to, finally, justify the claim that inferences peformed according to erotetic rules can be viewed as valid inferences.

Definition 8.1. (partitions of a language $\mathcal{L}_{-\mathrm{L}}^{\text {? }}$ ). A partition of $\mathcal{D}_{\mathcal{L}_{\dashv L}}$ is an ordered pair:

$$
P=\left\langle T_{P}, U_{P}\right\rangle
$$

where $T_{P} \cap U_{P}=\emptyset$ and $T_{P} \cup U_{P}=\mathcal{D}_{\mathcal{L}_{\overrightarrow{\lrcorner}}^{?}}$.

In the sequel we will use the notion of L-admissible partition, which is relative to a given logic $\mathrm{L}$.

Definition 8.2. (CPL-admissible partitions of $\mathcal{L}_{-\mathrm{CPL}}^{\text {? }}$ ). A partition $P=$ $\left\langle T_{P}, U_{P}\right\rangle$ of $\mathcal{L}_{-}^{\text {?CPL }}$ is CPL-admissible iff the following conditions hold:

1. $\left\ulcorner\dashv S^{\prime} \alpha^{\prime} T\right\urcorner \in T_{P}$ iff $\left\ulcorner\dashv S^{\prime} \alpha_{1}{ }^{\prime} \alpha_{2}{ }^{\prime} T\right\urcorner \in T_{P}$

2. $\left\ulcorner\dashv S^{\prime} \beta^{\prime} T\right\urcorner \in T_{P}$ iff $\left\ulcorner\dashv S^{\prime} \beta_{1}{ }^{\prime} T\right\urcorner \in T_{P}$ or $\left\ulcorner\dashv S^{\prime} \beta_{2}{ }^{\prime} T\right\urcorner \in T_{P}$

3. $\left\ulcorner\dashv S^{\prime} \neg \neg A^{\prime} T\right\urcorner \in T_{P}$ iff $\left\ulcorner\dashv S^{\prime} A^{\prime} T\right\urcorner \in T_{P}$

4. if $\left\ulcorner\dashv \underline{S}^{\prime} \underline{T}^{\prime} \underline{U}^{\prime} \underline{V}\right\urcorner \in T_{P}$, then $\left\ulcorner\dashv S^{\prime} A^{\prime} T\right\urcorner \in T_{P}$ or $\left\ulcorner\dashv U^{\prime} \bar{A}^{\prime} V\right\urcorner \in T_{P}$

5. $\ulcorner(\mathfrak{r} \sqcup \mathfrak{u})\urcorner \in T_{P}$ iff $\mathfrak{r} \in T_{P}$ or $\mathfrak{u} \in T_{P}$

6. $\mathfrak{u} \in T_{P}$ iff $\ulcorner n g(\mathfrak{u})\urcorner \notin T_{P}$

Definition 8.3. (L-admissible partitions of $\mathcal{L}_{-\downarrow}^{\text {? }}$ for $L=C L u N, C L u N s, m b C$ ). In the case of $L=C L u N$ we add to clauses 1.-6. of Definition 8.2 the following items:

7. $\left\ulcorner\dashv S^{\prime} \sim A^{\prime} T\right\urcorner \in T_{P}$ iff $\left\ulcorner\dashv S^{\prime} \neg A^{\prime} T\right\urcorner \in T_{P}$ or $\left\ulcorner\dashv S^{\prime} \chi \sim A^{\prime} T\right\urcorner \in T_{P}$

8. $\left\ulcorner\dashv S^{\prime} \neg \sim A^{\prime} T\right\urcorner \in T_{P}$ iff $\left\ulcorner\dashv S^{\prime} A^{\prime} \neg \chi \sim A^{\prime} T\right\urcorner \in T_{P}$

In the case of $\mathrm{L}=$ CLuNs we add to 1.-8. the following clauses:

\footnotetext{
${ }^{9}$ This is a central notion of Minimal Erotetic Semantics (MiES, for short). An extensive analysis of MiES can be found in [27].
} 
9. $\left\ulcorner\dashv S^{\prime} * \alpha^{\prime} T\right\urcorner \in T_{P}$ iff $\left\ulcorner\dashv S^{\prime} * \alpha_{1}{ }^{\prime} * \alpha_{2}{ }^{\prime} T\right\urcorner \in T_{P}$

10. $\left\ulcorner\dashv S^{\prime} * \beta^{\prime} T\right\urcorner \in T_{P}$ iff $\left\ulcorner\dashv S^{\prime} * \beta_{1}{ }^{\prime} T\right\urcorner \in T_{P}$ or $\left\ulcorner\dashv S^{\prime} * \beta_{1}{ }^{\prime} T\right\urcorner \in T_{P}$

11. $\left\ulcorner\dashv S^{\prime} \sim \sim A^{\prime} T\right\urcorner \in T_{P}$ iff $\left\ulcorner\dashv S^{\prime} A^{\prime} T\right\urcorner \in T_{P}$

Finally, for the case of $L=m b C$ we add to $1 .-8$. the following items:

$$
\begin{aligned}
9^{*} . & \left\ulcorner\dashv S^{\prime} \circ A^{\prime} T\right\urcorner \in T_{P} \text { iff }\left\ulcorner\dashv S^{\prime} \neg A^{\prime} \chi \circ A^{\prime} T\right\urcorner \in T_{P} \text { or } \\
& \left\ulcorner\dashv S^{\prime} \neg \sim A^{\prime} \chi \circ A^{\prime} T\right\urcorner \in T_{P} \\
10^{*} . & \left\ulcorner\dashv S^{\prime} \neg \circ A^{\prime} T\right\urcorner \in T_{P} \text { iff }\left\ulcorner\dashv S^{\prime} A^{\prime} \sim A^{\prime} T\right\urcorner \in T_{P} \text { or } \\
& \left\ulcorner\dashv S^{\prime} \neg \chi \circ A^{\prime} T\right\urcorner \in T_{P}
\end{aligned}
$$

The notion of entailment between the d-wffs of $\mathcal{L}_{-L}^{?}$ is introduced in a generalized fashion, as the relation of the multiple-conclusion entailment, symbolized by ' $\|=$ ':

Definition 8.4. (multiple-conclusion entailment). Let $X$ and $Y$ stand for arbitrary sets of d-wffs of language $\mathcal{L}_{-\mathrm{L}}^{\text {? }} \cdot X \|_{\mathcal{L}_{\mathcal{H}}^{?}} Y$ iff there is no Ladmissible partition $P=\left\langle T_{P}, U_{P}\right\rangle$ of $\mathcal{L}_{\dashv \mathrm{L}}^{\text {? }}$ such that $X \subset T_{P}$ and $Y \subset U_{P}$.

Let us look at some examples:

$$
\begin{aligned}
& \{\ulcorner\dashv \neg \neg A\urcorner\} \|=_{\mathcal{L}_{\dashv \mathrm{CPL}}^{?}}\{\ulcorner\dashv A\urcorner\} \\
& \{\ulcorner\dashv \neg \circ A\urcorner\} \|=_{\mathcal{L}_{\dashv \mathrm{mbc}}^{?}}\left\{\left\ulcorner\dashv A^{\prime} \sim A\right\urcorner,\ulcorner\dashv \neg \chi \circ A\urcorner\right\} \\
& \{\ulcorner\dashv \neg \circ A\urcorner\}\|\|_{\mathcal{L}_{\dashv \mathrm{mbc}}^{?}}\left\{\left\ulcorner\left(\dashv A^{\prime} \sim A\right) \sqcup(\dashv \neg \chi \circ A)\right\urcorner\right\}
\end{aligned}
$$

Finally, the patient reader may check that the following holds:

Theorem 8.5. Let $Q=?\left(\phi_{1}, \ldots, \phi_{n}\right)$ and $Q^{*}=?\left(\psi_{1}, \ldots, \psi_{m}\right)$ be questions of $\mathcal{L}_{{ }_{\mathrm{H}}}^{?}$. If $Q^{*}$ results from $Q$ by an application of a rule of $\mathbb{E}_{\text {res }}^{\mathrm{L}}$, then

1. $\left\{\phi_{1} \sqcup \ldots \sqcup\left(\phi_{n-1} \sqcup \phi_{n}\right)\right\} \|=\left\{\psi_{1} \sqcup \ldots \sqcup\left(\psi_{m-1} \sqcup \psi_{m}\right)\right\}$

2. $\left\{\psi_{1} \sqcup \ldots \sqcup\left(\psi_{m-1} \sqcup \psi_{m}\right)\right\} \|=\left\{\phi_{1} \sqcup \ldots \sqcup\left(\phi_{n-1} \sqcup \phi_{n}\right)\right\}$

3. $\left\{n g\left(\phi_{1} \sqcup \ldots \sqcup\left(\phi_{n-1} \sqcup \phi_{n}\right)\right)\right\} \|=\left\{n g\left(\psi_{1} \sqcup \ldots \sqcup\left(\psi_{m-1} \sqcup \psi_{m}\right)\right)\right\}$

4. $\left\{n g\left(\psi_{1} \sqcup \ldots \sqcup\left(\psi_{m-1} \sqcup \psi_{m}\right)\right)\right\} \|=\left\{n g\left(\phi_{1} \sqcup \ldots \sqcup\left(\phi_{n-1} \sqcup \phi_{n}\right)\right)\right\}$

The above theorem shows that when one arrives at $Q^{*}$ by a reformulation of $Q$ in guidance with the erotetic rules, then the affirmative answers to $Q$ and $Q^{*}$ entail each other (in a well-defined sense), and the negative answers to $Q$ and $Q^{*}$ entail each other. Whenever this is the case, one may say that the relations of erotetic implication or that of p-equipollence hold between these questions. Both these relations serve as fruitful explications of the intuitions we may assign to the validity of erotetic inferences. All the 
definitions of these interesting concepts may be found in works by Andrzej Wiśniewski (see $[25,27]$ ).

\section{Conclusions}

In this paper we have presented an original account of the resolution system for the paraconsistent logic $\mathrm{mbC}$, and also similar systems for the propositional parts of paraconsistent logics CLuN and CLuNs. The resolution rule of our calculi is dual with respect to the standard one (e.g. it acts on dual clauses, i.e., conjunctions of formulas) and is non-clausal.

The resolution systems presented in this paper form the "skeletons" of the so-called erotetic calculi - calculi of questions which allow us to simplify the logical structure of a certain problem expressed by a question, and possibly to solve the problem (and thus answer the question) purely by transforming questions. The method of transforming questions concerning the consequence relation of a logic $L$ according to the rules of an erotetic calculus is called the method of Socratic proofs for $\mathrm{L}$.

The method of Socratic proofs is grounded in the logic of questions IEL and it may be shown that there is a semantic counterpart of the relation generated by the erotetic rules. Thus the rules of erotetic calculi constitute a model of valid erotetic paraconsistent inferences.

Acknowledgements. This work has been supported by the Polish National Science Center, grant no. 2012/04/A/HS1/00715. We are indebted to Andrzej Wiśniewski, Mariusz Urbański and Paweł Łupkowski for sharing and discussing ideas.

Open Access. This article is distributed under the terms of the Creative Commons Attribution 4.0 International License (http://creativecommons. org/licenses/by/4.0/), which permits unrestricted use, distribution, and reproduction in any medium, provided you give appropriate credit to the original author(s) and the source, provide a link to the Creative Commons license, and indicate if changes were made.

\section{References}

[1] Avron, A., The method of hypersequents in the proof theory of propositional nonclassical logics, in W. Hodges, M. Hyland, C. Steinhorn, and J. Truss, (eds.), Logic: Foundations to Applications, Oxford Science Publications, New York, 1996, pp. 1-32.

[2] Batens, D., Paraconsistent extensional propositional logics, Logique et Analyse 9091:195-234, 1980. 
[3] Batens, D., Inconsistency-adaptive logics, in E. Orłowska, (ed.), Logic at Work. Essays Dedicated to the Memory of Helena Rasiowa, Springer Verlag, Heidelberg, 1998, pp. 445-472.

[4] Batens, D., and K. De ClercQ, A rich paraconsistent extension of full positive logic, Logique et Analyse 185-188:227-257, 2005.

[5] Batens, D., K. De Clercq, and N. Kurtonina, Embedding and interpolation for some paralogics. The propositional case, Reports on Mathematical Logic 33:29-44, 1999.

[6] Batens, D., and J. Meneus, A tableau method for inconsistency-adaptive logics, in R. Dyckhoff, (ed.), Automated Reasoning with Analytic Tableaux and Related Methods, Springer, Dordrecht, 2000, pp. 127-142.

[7] Carnielli, W. A., and M. E. Coniglio, Logics of formal inconsistency, in F. Guenthner and Dov M. Gabbay, (eds.), Handbook of Philosophical Logic, vol. 14, Springer, Dordrecht, 2013, pp. 1-93.

[8] Carnielli, W. A., and J. Marcos, A taxonomy of $\mathbf{C}$-systems, in I. $\mathbf{M}$. L. D'Ottaviano, W. A. Carnielli, and M. E. Coniglio, (eds.), Paraconsistency-The Logical Way to the Inconsistent, Marcel Dekker, New York, 2000, pp. 1-94.

[9] Coniglio, M. E., and T. G. Rodrigues, Some investigations on $\mathrm{mbC}$ and $\mathrm{mCi}$, in C. A. Mortari, (ed.), Tópicos de lógicas não clássicas, NEL/UFSC, Florianópolis, 2014, pp. 11-70.

[10] Fitting, M., First-Order Logic and Automated Theorem Proving, Springer Verlag, Berlin, 1990.

[11] Indrzejczak, A., Natural Deduction, Hybrid Systems and Modal Logics, vol. 30 of Trends in Logic, Springer, Dordrecht, 2010.

[12] Indrzejczak, A., A survey of nonstandard sequent calculi, Studia Logica 102(6):1295-1322, 2014.

[13] LesZCZyŃskA, D., Socratic proofs for some normal modal propositional logics, Logique et Analyse 47(185-188):259-285, 2004.

[14] Leszczyńska-Jasion, D., The Method of Socratic Proofs for Normal Modal Propositional Logics, Adam Mickiewicz University Press, Poznań, 2007.

[15] Leszczyńska-Jasion, D., The method of Socratic proofs for modal propositional logics: K5, S4.2, S4.3, S4M, S4F, S4R and G, Studia Logica 89(3):371-405, 2008.

[16] Leszczyńska-Jasion, D., M. URbański, and A. Wiśniewski, Socratic trees, Studia Logica 101(5):959-986, 2013.

[17] LigezA, A., Logical Foundations for Rule-Based Systems, vol. 11 of Studies in Computational Intelligence, Springer, Heidelberg, 2006.

[18] Negri, S., and J. von Plato, Structural Proof Theory, Cambridge University Press, Cambridge, 2001.

[19] Neto, A. G. S. S., and M. Finger, Effective prover for minimal inconsistency logic, in M. Bramer, (ed.), IFIP International Federation for Information Processing, Springer, New York, 2006, pp. 465-474.

[20] Robinson, J. A., A machine oriented logic based on the resolution principle, Journal of the ACM 12:23-41, 1965. 
[21] Skura, T. F., Intuitionistic Socratic procedures, Journal of Applied Non-Classical Logics 15(4):453-464, 2005.

[22] SkurA, T. F., Refutation systems in propositional logic, in F. Guenthner and D. M. Gabbay, (eds.), Handbook of Philosophical Logic, vol. 16, Springer, New York, 2011, pp. 115-157.

[23] Smullyan, R. M., First-Order Logic, Springer-Verlag, Berlin, 1968.

[24] Troelstra, A. S., and H. Schwichtenberg, Basic Proof Theory, 2nd edn., Camridge University Press, Cambridge, 2000.

[25] Wiśniewski, A., The Posing of Questions: Logical Foundations of Erotetic Inferences, Kluwer Academic Publishers, Dordrecht, 1995.

[26] Wiśniewski, A., Socratic proofs, Journal of Philosophical Logic 33(3):299-326, 2004.

[27] Wiśniewski, A., Questions, Inferences and Scenarios, vol. 46 of Studies in Logic. Logic and Cognitive Systems, College Publications, London, 2013.

[28] Wiśniewski, A., and V. Shangin, Socratic proofs for quantifiers, Journal of Philosophical Logic 35(2):147-178, 2006.

[29] Wiśniewski, A., and V. Shangin, Некоторые допустимые правила дла системы сократического вывода (Some admissible rules in the system of Socratic proofs), Вестник Московского Университета. Серия 7(5):77-88, 2007.

[30] Wiśniewski, A., G. Vanackere, and D. Leszczyńska, Socratic proofs and paraconsistency: A case study, Studia Logica 80(2-3):433-468, 2005.

Sz. Chlebowski, D. Leszczyńska-Jasion

Department of Logic and Cognitive Science

Institute of Psychology

Adam Mickiewicz University

Szamarzewskiego 89a, 60-568

Poznań, Poland

szymon.chlebowski@amu.edu.pl

D. LESZCZYŃSKA-JASION

dorota.leszczynska@amu.edu.pl 